TRANSACTIONS OF THE

AMERICAN MATHEMATICAL SOCIETY

Volume 277, Number 1, May 1983

\title{
CONFORMALLY INVARIANT VARIATIONAL INTEGRALS
}

BY

S. GRANLUND, P. LINDQVIST AND O. MARTIO

\begin{abstract}
Let $f: G \rightarrow R^{n}$ be quasiregular and $I=\int F(x, \nabla u) d m$ a conformally invariant variational integral. Hölder-continuity, Harnack's inequality and principle are proved for the extremals of $I$. Obstacle problems and their connection to subextremals are studied. If $u$ is an extremal or a subextremal of $I$, then $u \circ f$ is again an extremal or a subextremal if an appropriate change in $F$ is made.
\end{abstract}

1. Introduction. A mapping $f: G \rightarrow R^{n}, G$ open in $R^{n}$, is called quasiregular if $f$ is continuous and $A C L^{n}$ in $G$ with $\left|f^{\prime}(x)\right|^{n} \leqslant K J(x, f)$ a.e. in $G$ for some $K \geqslant 1$. A homeomorphic quasiregular mapping onto $f G$ is called quasiconformal. If $n=2$ and $K=1, f=u_{1}+i u_{2}$ is analytic or conformal, respectively. The functions $u_{1}$ and $u_{2}$ are harmonic functions and hence extremals for the Dirichlet-integral. The coordinate functions $f_{1}, \ldots, f_{n}$ of a quasiregular mapping $f: G \rightarrow R^{n}$ are extremals of the variational integral $\int F(x, \nabla u) d m$ where $F(x, h) \approx|h|^{n}$ and $F$ depends on $f$. It is well known that the Dirichlet-integral remains invariant under conformal mappings $f$ of the plane domain $G$, i.e.

$$
\int_{f G}|\nabla u|^{2} d m=\int_{G}|\nabla(u \circ f)|^{2} d m .
$$

In space and in plane it is possible to rephrase this equality for general kernels $F$ and for quasiconformal mappings when an appropriate change in $F$ is made. For analytic and quasiregular mappings this invariance property has the counterpart which takes into account the many-to-one character of these mappings. The capacity inequalities of quasiconformal and quasiregular mappings, see [G, MRV1 and M1], form a special case of this invariance property. This phenomenon can also be studied using partial differential equations, the Euler equations of the corresponding variational integrals, and the basic fact is that harmonic functions in plane remain harmonic under composition with analytic functions. Yu. G. Rešetnjak [R4] has studied the corresponding property of quasiregular mappings. A new proof for this result is presented in a more general case.

Subharmonic functions form a natural generalization of harmonic functions. The main purpose of this paper is to show that this class of functions has a generalization to the nonlinear case in space as well. We call these functions sub- $F$-extremals since for our purposes the extremality property expressed in terms of variational integrals

Received by the editors September $9,1981$.

1980 Mathematics Subject Classification. Primary 30C70; Secondary 49A21, 35A15, 31B99.

Key words and phrases. Variational integrals, quasiregular mappings, subextremals. 
is essential. If $u$ is subharmonic and $f$ analytic in plane, then it is well known that $u \circ f$ is subharmonic. The proof of this fact is usually based on the submean property of subharmonic functions and the Taylor-expansion of analytic functions, see [HK, p. 53]. It can also be derived using smooth subharmonic approximations of a general subharmonic function. These methods are not directly available in the general case and we present a proof for the invariance property of sub- $F$-extremals based on certain integral inequalities. This principle clearly has an effect on the theory of quasiregular mappings and the authors intend to return to these problems in subsequent papers.

The paper is organized as follows. After preliminaries in $\$ 2$ variational integrals and their extremals are introduced in $\S 3$. The connection to differential equations is studied in detail and the existence of continuous extremals up to the boundary is proved. Here we use a simple compactness property introduced in [M2] and the lower semicontinuity of variational integrals, especially no advanced Sobolev space analysis on the boundary is needed. In $\$ 4$ properties of extremals are studied. In the case $F(x, h) \approx|h|^{n}$ we present new and simple proofs for the Hölder-continuity and Harnack's inequality of the extremals, cf. [Gr, Li2]. These proofs are based on F. W. Gehring's oscillation lemma. Moreover, these methods give effective numerical bounds for the various exponents and constants involved. $\S 5$ is devoted to sub- $F$ extremals. A sub-F-extremal $u$ is called regular if $u$ is $A C L^{n}$. It turns out that this corresponds to the class of plane $C^{2}$-subharmonic functions and integral- and differential inequalities characterizing this class are presented. $\$ 6$ deals with the invariance of integrals under quasiconformal and quasiregular mappings. The invariance of extremals, sub- $F$-extremals and regular sub- $F$-extremals is then proved in $\$ 7$.

The paper contains some new methods not previously employed in the calculus of variations. The method of $\mathrm{H}$. Lebesgue, the use of monotone functions, is extended to obstacle problems under minimal assumptions on the kernel $F$ in $\S 5$. The Schwarz alternating process is applied to the general case in the same section. The fact that the conformally invariant situation deals with the borderline case $F(x, h) \approx|h|^{n}$ is used to the full extent. Our methods cannot be used for $F(x, h) \approx|h|^{p}, 1<p<n$, in the same generality and we have tried to indicate those places where $p=n$ is needed.

\section{Preliminaries.}

2.1. Notation. For each set $A$ in the euclidean space $R^{n}$ we let $C A, \bar{A}$, and $\partial A$ denote the complement, closure, and boundary of $A$, all taken with respect to $R^{n}$. Given two sets $A \subset B$ in $R^{n}, A \subset \subset B$ means that $\bar{A}$ is compact in $B$. By $x \cdot y$ we denote the usual inner product of two vectors $x$ and $y$ in $R^{n}$. For $x \in R^{n}$ we use representations $x=\left(x_{1}, \ldots, x_{n}\right)=\sum x_{i} e_{i}$. If $x \in R^{n}$ and $r>0$, we let $B^{n}(x, r)$ denote the open ball $\left\{y \in R^{n}:|y-x|<r\right\}$, and $S^{n-1}(x, r)$ is the sphere $\partial B^{n}(x, r)$. We shall employ the abbreviations $B^{n}(r)=B^{n}(0, r), B^{n}=B^{n}(1), S^{n-1}(r)=$ $S^{n-1}(0, r), S^{n-1}=S^{n-1}(1)$.

The Lebesgue measure of a set $A \subset R^{n}$ will be written as $m(A)$. The normalized $(n-1)$-measure in $(n-1)$-dimensional spheres is written as $S$. We set $\omega_{n-1}=$ $S\left(S^{n-1}\right)$. 
If $A \subset R^{n}$, then $C(A)$ is the class of all continuous (real valued) functions on $A$. If $U$ is an open set in $R^{n}$, we let $C^{p}(U)$ denote the family of all $p$ times continuously differentiable functions $u: U \rightarrow R$, and $C_{\delta}(U)$ the family of all $u \in C^{p}(U)$ whose support spt $u$ is a compact subset of $U$. The classes $C^{p}(A)$ are also defined for smooth submanifolds $A$ of $R^{n}$.

2.2. Sobolev space $W_{p}^{1}$ and $A C L^{p}$-functions. If $A$ is a Lebesgue measurable subset of $R^{n}$, then $L^{p}(A), p \geqslant 1$, is the Banach space of all functions $u$ : $A \rightarrow \dot{R}=R \cup$ $\{-\infty, \infty\}$ with the norm

$$
\|u\|_{p}=\|u\|_{p, A}=\left(\int_{A}|u|^{p} d m\right)^{1 / p} .
$$

We also use the same expression for functions $u: A \rightarrow R^{m}$, i.e. $\|u\|_{p}=\|u\|_{p}$. Given an open set $G$ in $R^{n}, W_{p}^{1}(G)$ is the Sobolev space of functions $u \in L^{p}(G)$ whose first distributional partial derivatives $D_{i} u$ belong to $L^{p}(G)$ with the norm

$$
\|u\|_{1, p}=\|u\|_{p}+\|\nabla u\|_{p} .
$$

Here $\nabla u=\left(D_{1} u, \ldots, D_{n} u\right)$ is the gradient of $u$. The space $W_{p, 0}^{1}(G)$ is the closure of $C_{0}^{\infty}(G)$ in $W_{p}^{1}(G)$.

A mapping $f: G \rightarrow R^{m}$ is said to be $A C L$ if $f$ is continuous and if for each open $n$-interval $Q \subset \subset G, f$ is absolutely continuous on almost every line segment in $\bar{Q}$, parallel to the coordinate axes. An $A C L$-mapping has partial derivatives a.e. If these are locally $L^{p}$-integrable, $p \geqslant 1, f$ is said to be $A C L^{p}$. It is well known that $f: G \rightarrow R$ is $A C L^{p}$ if and only if $f$ is continuous and belongs to $W_{p}^{1}(D)$ for each open set $D \subset \subset G$. For basic properties of Sobolev spaces and $A C L$-functions see [Mor].

2.3. Condensers and their capacity. A condenser in $R^{n}$ is a pair $E=(A, C)$ where $A \subset R^{n}$ is open and $C$ is a nonempty compact set contained in $A$. We set for $p \geqslant 1$

$$
\operatorname{cap}_{p} E=\operatorname{cap}_{p}(A, C)=\inf _{u \in W_{0}(E)} \int_{A}|\nabla u|^{p} d m
$$

and call it the $p$-capacity of the condenser $E$. The set $W_{0}(E)=W_{0}(A, C)$ is the family of all nonnegative functions $u \in C_{0}(A)$ which are $A C L$ and satisfy $u(x)=1$ for $x \in C$. It is well known that $W_{0}(E)$ can be replaced in (2.4) by $W_{0}(E) \cap C_{0}^{\infty}(A)$. We are mainly interested in the case $p=n$. Note that if $\rho>r>0$, then

$$
\operatorname{cap}_{n}\left(B^{n}\left(x_{0}, \rho\right), \bar{B}^{n}\left(x_{0}, r\right)\right)=\omega_{n-1}(\ln (\rho / r))^{1-n} .
$$

2.5. Gehring's oscillation lemma. If $A \subset R^{n}$ is a set and $u: A \rightarrow R$ we let $\omega(u, A)=\sup _{A} u-\inf _{A} u$ denote the oscillation of $u$ on $A$.

For $x \in S^{n-1}(r), n \geqslant 2$, and for $\varphi \in(0, \pi]$ let

$$
C(x, \varphi)=\left\{y \in S^{n-1}(r): y \cdot x>r^{2} \cos \varphi\right\} .
$$

Then $C(x, \varphi)$ is a spherical cap (possibly a punctured sphere) on $S^{n-1}(r)$ centered at $x$. The following lemma was proved for $n=3$ in [G, Lemma 1] and for arbitrary $n \geqslant 3$ in [Mos] when $C(x, \varphi)=S^{n-1}(r)$. The case $n=2$ is trivial and for $C(x, \varphi)$ instead of $S^{n-1}(r)$ the proof is completely analogous. 
2.6. Lemma. Suppose that $u \in C^{1}(C(x, \varphi))$. Then

$$
\omega(u, C(x, \varphi))^{n} / r \leqslant A_{n} \int_{C(x, \varphi)}\left|\nabla_{s} u\right|^{n} d S
$$

and $A_{n}<\infty$ depends only on $n$. Here $\nabla_{s} u$ is the gradient of $u$ on $S^{n-1}(r)$.

We shall frequently consider the following situation. Let $G$ be open in $R^{n}$ and $u$ an $A C L^{n}$-function in $G$. Suppose that for $r \in\left(r_{1}, r_{2}\right)$ the spherical caps $C\left(x_{r}, \varphi_{r}\right)$, $x_{r} \in S^{n-1}(r)$, lie in $G$. If the function $r \mapsto \omega\left(u, C\left(x_{r}, \varphi_{r}\right)\right)$ is measurable in the interval $\left(r_{1}, r_{2}\right)$, then by approximation and by Fubini's theorem Lemma 2.6 yields

\subsection{LEMMA.}

$$
\int_{r_{1}}^{r_{2}} \omega\left(u, C\left(x_{r}, \varphi_{r}\right)\right)^{n} / r d r \leqslant A_{n} \int_{G}|\nabla u|^{n} d m .
$$

Proof. If $\|\nabla u\|_{n}=\infty$, there is nothing to prove. Suppose that $\|\nabla u\|_{n}<\infty$. By Fubini's theorem and by the invariance of the $A C L^{n}$-property under smooth transformations for a.e. $r \in\left(r_{1}, r_{2}\right), \int_{S^{n-1}(r) \cap G}|\nabla u|^{n} d S<\infty$ and the function $u$ is $A C L$ in $S^{n-1}(r) \cap G$. Observe that via the stereographic projection of $S^{n-1}(r)$ into $R^{n-1}$ the $A C L$-property in $S^{n-1}(r)$ becomes the ordinary $A C L$-property in $R^{n-1}$. By approximation Lemma 2.6 yields for a.e. $r \in\left(r_{1}, r_{2}\right)$

$$
\omega\left(u, C\left(x_{r}, \varphi_{r}\right)\right)^{n} / r \leqslant A_{n} \int_{S^{n-1}(r) \cap G}\left|\nabla_{s} u\right|^{n} d S \leqslant A_{n} \int_{S^{n-1}(r) \cap G}|\nabla u|^{n} d S
$$

and the result follows by integration.

2.8. Monotone functions. Let $G$ be an open set in $R^{n}$. A function $u \in C(G)$ is called monotone (in the sense of Lebesgue) if for each domain $D \subset \subset G$ we have $\omega(u, D)=$ $\omega(u, \partial D)$. If $G$ is bounded and $u \in C(\bar{G})$, the condition $D \subset \subset G$ may be replaced by $D \subset G$.

2.9. Regular open sets. Instead of various conditions for Dirichlet-regular domains, see 3.25(a), we shall employ a topological definition for regularity. This condition is adequate for our purposes and satisfied in most applications. An open set $G \subset R^{n}$ is called regular if it is bounded and $\partial G$ contains no point components.

In [M2], the following equicontinuity lemma was proved for monotone functions under a less restrictive condition on $\partial G$.

2.10. LemMA. Suppose that $G \subset R^{n}$ is regular and $v \in C(\bar{G})$. Let $\mathscr{F}$ be a family of functions $u \in C(\bar{G}) \cap W_{n}^{1}(G)$ such that

$$
\omega(u, D) \leqslant \max (\omega(u, \partial D), \omega(v, D)) \text { for each domain } D \subset G
$$

and

$$
\int_{G}|\nabla u|^{n} d m \leqslant M \quad \text { for all } u \in \mathcal{F} .
$$

Then $\mathscr{F}$ is equicontinuous if and only if $\mathscr{F} \mid \partial G$ is equicontinuous.

Proof. It suffices to show that the equicontinuity of $\mathscr{F} \mid \partial G$ implies the equicontinuity of $\mathscr{F}$. We first prove that this holds at a point $x_{0} \in G$. We may assume that $x_{0}=0$. Let $\varepsilon>0$. Choose $\rho>0$ such that $B^{n}(\rho) \subset G$ and $\omega\left(v, B^{n}(\rho)\right)<\varepsilon$. Let 
$r \in(0, \rho)$ and $u \in \mathscr{F}$. Pick $t \in[r, \rho]$ with $\omega\left(u, S^{n-1}(t)\right)=\min \left\{\omega\left(u, S^{n-1}(s)\right): s \in\right.$ $[r, \rho]\}$. Then Lemma 2.7 yields

$$
\begin{aligned}
\omega\left(u, S^{n-1}(t)\right)^{n} \log \rho / r & \leqslant \int_{r}^{\rho} \omega\left(u, S^{n-1}(s)\right)^{n} / s d s \\
& \leqslant A_{n} \int_{G}|\nabla u|^{n} d m \leqslant A_{n} M .
\end{aligned}
$$

Thus there is $r \in(0, \rho)$, independent of $u \in \mathcal{F}$, such that $\omega\left(u, S^{n-1}(t)\right)<\varepsilon$. Consequently, by (2.11),

$$
\omega\left(u, B^{n}(r)\right) \leqslant \omega\left(u, B^{n}(t)\right) \leqslant \max \left(\omega\left(u, S^{n-1}(t)\right), \omega\left(v, B^{n}(t)\right)\right)<\varepsilon .
$$

This shows the equicontinuity of $\mathscr{F}$ at $x_{0} \in G$.

Next consider a point $x_{0} \in \partial G$. We may again assume $x_{0}=0$. Suppose that $\mathscr{F}$ is not equicontinuous at 0 . Then there is $\varepsilon>0$ such that for all $t>0$ there is $u=u_{t} \in \mathscr{F}$ with

$$
\omega(u, G(t))>\varepsilon
$$

where $G(t)=\bar{G} \cap \bar{B}^{n}(t)$. Since $G$ is regular and $\mathscr{F} \mid \partial G$ is equicontinuous, there is $\rho>0$ such that

$$
S^{n-1}(t) \cap \partial G \neq \varnothing
$$

for all $t \in(0, \rho]$ and

$$
\omega\left(u, \partial G \cap \bar{B}^{n}(\rho)\right)<\varepsilon / 16
$$

for all $u \in \mathscr{F}$ and, moreover,

$$
\omega(v, G(\rho))<\varepsilon / 8 .
$$

Fix $t \in(0, \rho)$ and let $u \in \mathscr{F}$ satisfy (2.13). Consider the function

$$
w(x)=\frac{16}{\varepsilon} \max (|u(x)-u(0)|-\varepsilon / 16,0) .
$$

Then $w \in C(\bar{G}) \cap W_{n}^{1}(G)$. Write $A=\{x \in G(\rho): w(x)>1\}$. Now $A=A_{1} \cup A_{2}$ where

$$
\begin{aligned}
& A_{1}=\{x \in G(\rho): u(x)>u(0)+\varepsilon / 8\}, \\
& A_{2}=\{x \in G(\rho): u(x)<u(0)-\varepsilon / 8\} .
\end{aligned}
$$

By (2.15), $A$ does not meet $\partial G$.

The set $\bar{A}$ meets $S^{n-1}(s)$ for all $s \in(t, \rho)$. To see this let $A(s)=A \cap B^{n}(s)$ and suppose that $A(s) \subset \subset B^{n}(s)$. If $D^{\prime}$ is a component of $A(s)$, then $\omega\left(u, \partial D^{\prime}\right)=0$ and thus

$$
\omega\left(u, D^{\prime}\right) \leqslant \max \left(\omega\left(u, \partial D^{\prime}\right), \omega\left(v, D^{\prime}\right)\right)<\varepsilon / 8
$$

by (2.11) and (2.16). Hence

$$
\begin{aligned}
\omega(u, G(t)) & \leqslant \omega(u, G(s)) \leqslant \omega(u, A(s))+\omega(u, G(s) \backslash A(s)) \\
& \leqslant \omega\left(u, A(s) \cap A_{1}\right)+\omega\left(u, A(s) \cap A_{2}\right)+\varepsilon / 4+\varepsilon / 4 \\
& <\varepsilon / 8+\varepsilon / 8+\varepsilon / 4+\varepsilon / 4=3 \varepsilon / 4,
\end{aligned}
$$

which is impossible by (2.13). 
For each $s \in(t, \rho)$ choose a cap $C\left(x_{s}, \varphi_{s}\right), \varphi_{s} \in(0, \pi]$, on $S^{n-1}(s) \cap G$ such that $w\left(x_{s}\right)=\sup \left\{w(x): x \in S^{n-1}(s) \cap \bar{G}\right\}$ and $\bar{C}\left(x_{s}, \varphi_{s}\right)$ meets $\partial G$. Then by (2.15), $\omega\left(w, C\left(x_{s}, \varphi_{s}\right)\right) \geqslant 1$. Since $s \mapsto \omega\left(w, C\left(x_{s}, \varphi_{s}\right)\right)$ is continuous, Lemma 2.7 yields

$$
\begin{aligned}
\log \rho / t & \leqslant \int_{t}^{\rho} \omega\left(w, C\left(x_{s}, \varphi_{s}\right)\right)^{n} / s d s \leqslant A_{n} \int_{G}|\nabla w|^{n} d m \\
& \leqslant A_{n}(16 / \varepsilon)^{n} \int_{G}|\nabla u|^{n} d m \leqslant A_{n} M(16 / \varepsilon)^{n} .
\end{aligned}
$$

Note that $|\nabla w| \leqslant 16|\nabla u| / \varepsilon$ a.e. in $G$. The right-hand side is independent of $t$. Letting $t \rightarrow 0$ gives a contradiction. This completes the proof.

If we choose $v=0$ in $G$, Lemma 2.10 gives the following corollary, cf. [M2].

2.17. Corollary. Suppose that $G$ is a regular domain and that $\mathscr{F}$ is a family of monotone functions $u \in C(\bar{G}) \cap W_{n}^{1}(G)$ satisfying (2.12). Then $\mathcal{F}$ is equicontinuous if and only if $\mathcal{F} \mid \partial G$ is equicontinuous.

3. Variational integrals. Basic properties of conformally invariant variational integrals are introduced. We close with the existence of extremals.

3.1. Variational kernels. Although we are mainly interested in the case $p=n$, the assumptions (a)-(c) on a variational kernel $F$ are adequate for some auxiliary considerations.

Let $G$ be an open set in $R^{n}$ and let $F: G \times R^{n} \rightarrow R$ satisfy the assumptions:

(a) For each open set $D \subset \subset G$ and $\varepsilon>0$ there is a compact set $C \subset D$ with $m(D \backslash C)<\varepsilon$ and $F \mid C \times R^{n}$ is continuous.

(b) For a.e. $x \in G$ the function $h \mapsto F(x, h)$ is convex.

(c) There are constants $0<\alpha \leqslant \beta<\infty$ and $1<p<\infty$ such that for a.e. $x \in G$

$$
\alpha|h|^{p} \leqslant F(x, h) \leqslant \beta|h|^{p} \quad \text { for all } h \in R^{n} .
$$

The constant $p$ determines the type of $F$. The greatest $\alpha \leqslant 1$, resp. the smallest $\beta \geqslant 1$, for which (3.2) holds is denoted by $\alpha(F)$, resp. $\beta(F)$. The numbers $\alpha(F)$ and $\beta(F)$ are called the structure constants of $F$.

In the connection of some uniqueness problems we shall replace (b) by a stronger condition:

(b') For a.e. $x \in G$ the function $h \mapsto F(x, h)$ is strictly convex, i.e.

$$
F\left(x, t h_{1}+(1-t) h_{2}\right)<t F\left(x, h_{1}\right)+(1-t) F\left(x, h_{2}\right)
$$

for $0<t<1$ and $h_{1}, h_{2} \in R^{n}, h_{1} \neq h_{2}$.

To connect variational problems with the solutions of the corresponding Euler-equations we use the assumption:

(d) For a.e. $x \in G$ the function $h \mapsto F(x, h)$ is differentiable.

Since, by (b), the function $h \mapsto F(x, h)$ is convex a.e. in $G$, we may as well in (d) assume that $h \mapsto F(x, h)$ is continuously differentiable, see [Ro, Corollary 25.5.1]. It turns out that condition (d) is not essential to our theory, however, its omission will give technical difficulties. In all interesting cases, condition (d) is satisfied in a natural way. 
Finally, to obtain a conformally invariant class we introduce the homogeneity condition:

(e) For a.e. $x \in G, F(x, \lambda h)=|\lambda|^{p} F(x, h)$ for all $\lambda \in R$ and $h \in R^{n}$.

3.3. REMARK. If $h: G \rightarrow R^{n}$ is a measurable function, then the function $x \mapsto$ $F(x, h(x))$ is measurable. This follows from (a), since there is a Borel-partition $G=\cup_{i=0}^{\infty} A_{i}$. such that $m\left(A_{0}\right)=0$ and $F \mid A_{i} \times R^{n}, i=1,2, \ldots$, is continuous. Define $\Phi: G \rightarrow G \times R^{n}$ as $\Phi(x)=(x, h(x))$. Then $\Phi$ is measurable. If $B$ is open in $R$, then

$$
\Phi^{-1} F^{-1} B=\bigcup_{i=0}^{\infty} \Phi^{-1}\left(\left(A_{i} \times R^{n}\right) \cap F^{-1} B\right) .
$$

Since $\left(A_{i} \times R^{n}\right) \cap F^{-1}(B)$ is a Borel set, $i=1,2, \ldots$, and

$$
m\left(\Phi^{-1}\left(\left(A_{0} \times R^{n}\right) \cap F^{-1} B\right)\right)=0,
$$

the set $\Phi^{-1} F^{-1} B$ is measurable.

Note also that if $h$ is as above and $F$ satisfies the assumptions (a)-(d), then the function $x \mapsto \nabla_{h} F(x, h(x))$ is measurable. For this observe that since $F \mid A_{i} \times R^{n}$, $i=1,2, \ldots$, is continuous, the mapping $\nabla_{h} F \mid A_{i} \times R^{n}$ is a Borel function and the conclusion follows as above.

3.4. RemarK. Yu. Rešetnjak studied in [R3] kernels $F_{\theta}(x, h)=(\theta(x) h \cdot h)^{n / 2}$ where $\theta: G \rightarrow \mathrm{GL}\left(R^{n}, R^{n}\right)$ is a Borel map such that for all $x \in G$ the linear map $\theta(x)$ is selfadjoint and there exist constants $0<\alpha \leqslant \beta<\infty$ with

$$
\alpha|h|^{2} \leqslant \theta(x) h \cdot h \leqslant \beta|h|^{2}
$$

for a.e. $x \in G$ and all $h \in R^{n}$. It is easy to see that the kernels $F_{\theta}$ satisfy the assumptions (a), ( $\left.\mathrm{b}^{\prime}\right),(\mathrm{c})-(\mathrm{e})$ for $p=n$.

If $F$ satisfies (a) $-(\mathrm{d})$, then $\nabla_{h} F$ has an additional property which will be frequently used in the sequel.

3.5. Lemma. Suppose that $F: G \times R^{n} \rightarrow R$ satisfies (a)-(d). Then there is $\gamma<\infty$ such that for a.e. $x \in G$

$$
\left|\nabla_{h} F(x, h)\right| \leqslant \gamma|h|^{p-1}
$$

for all $h \in R^{n}$. The constant $\gamma$ depends only on $n, p$, and $\beta(F)$.

Proof. Fix $x \in G$ such that (b), (c), and (d) hold. Let $h \in R^{n}$. Choose $i$, $1 \leqslant i \leqslant n$, and let $\varphi(t)=F\left(x, h+t e_{i}\right)$. Then $\varphi: R \rightarrow R$ is convex and continuously differentiable. Since $\varphi^{\prime}(0)=D_{i} F(x, h)$, it suffices to show $\left|\varphi^{\prime}(0)\right| \leqslant 2^{p} \beta(F) \mid h^{p-1}$.

The convexity of $\varphi$ yields $\varphi(t)-\varphi(0) \geqslant \varphi^{\prime}(0) t$ for all $t$. On the other hand $\varphi$ is nonnegative, hence $\varphi(t) \geqslant \varphi^{\prime}(0) t$. By (c)

$$
\varphi(t) \leqslant \beta(F)\left|h+t e_{i}\right|^{p} \leqslant 2^{p-1} \beta(F)\left(|h|^{p}+|t|^{p}\right) .
$$

Thus

$$
\varphi^{\prime}(0) t \leqslant 2^{p-1} \beta(F)\left(|h|^{p}+|t|^{p}\right)
$$

for all $t$. Setting $t=\operatorname{sign}\left(\varphi^{\prime}(0)\right)|h|$ yields $\left|\varphi^{\prime}(0)\right| \leqslant 2^{p} \beta(F)|h|^{p-1}$, if $|h| \neq 0$. If $h=0,(3.6)$ is trivial by (c) since $p>1$. 
3.7. Variational integrals. Suppose that $F: G \times R^{n} \rightarrow R$ satisfies (a)-(c). If $u$ : $G \rightarrow R$ has partial derivatives a.e. or distributional derivatives in Sobolev's sense and $A \subset G$ is measurable, then there exists the variational integral

$$
I_{F}(u, A)=\int_{A} F(x, \nabla u(x)) d m(x),
$$

see Remark 3.3. If $A \subset \subset G$ and $u$ is $A C L^{p}$ in $G$ or $u \in W_{p}^{1}(G), I_{F}(u, A)$ is finite.

3.9. Lower semicontinuity. For the existence theory and various convergence problems the well-known lower semicontinuity property of (3.8) is useful. There exist many proofs for this result under different assumptions, see e.g. [Mor, R2]. For a very simple proof based on Banach-Saks' theorem under minimal assumptions see [Li1].

3.10. TheOREM. Suppose that $F$ satisfies (a)-(c) in an open set $G \subset R^{n}$. Let $u_{i}$, $i=0,1, \ldots$, be $W_{p}^{1}$-functions in $G$ and $\nabla u_{i} \rightarrow \nabla u_{0}$ weakly in $L^{p}(G)$. Then

$$
I_{F}\left(u_{0}, G\right) \leqslant \lim _{i \rightarrow \infty} I_{F}\left(u_{i}, G\right) .
$$

3.11. Remark. According to Banach-Saks' theorem there are indices $i_{1}<i_{2}<\cdots$ such that

$$
\left\|\left(\nabla u_{i_{1}}+\cdots+\nabla u_{i_{k}}\right) / k-\nabla u_{0}\right\|_{L^{p}(G)} \rightarrow 0 .
$$

Roughly speaking, this convergence feature, combined with Fatou's lemma and Jensen's inequality

$$
F\left(x,\left(\nabla u_{i_{1}}+\cdots+\nabla u_{i_{k}}\right) / k\right) \leqslant\left(F\left(x, \nabla u_{i_{1}}\right)+\cdots+F\left(x, \nabla u_{i_{k}}\right)\right) / k
$$

gives the desired result. Thus the only nontrivial part of the proof of Theorem 3.10 is the establishing of Banach-Saks' theorem in $L^{p}(G) \times \cdots \times L^{p}(G)$ ( $n$ times). However, this question is trivial in the Hilbert space $L^{2}(G) \times \cdots \times L^{2}(G)$ and in the borderline case $p=n \geqslant 2$ the proof can be reduced to the $L^{2}$-case, since $\nabla u_{i} \rightarrow \nabla u_{0}$ locally weakly in $L^{2}$ if $\nabla u_{i} \rightarrow \nabla u_{0}$ weakly in $L^{p}, p \geqslant 2$.

3.12. Extremals. Suppose that $F$ satisfies (a)-(c) in a bounded open set $G \subset R^{n}$ and $v \in C(\partial G)$. A function $u \in C(\bar{G}) \cap W_{p}^{1}(G), u=v$ in $\partial G$, is called an $F$ extremal with boundary values $v$ for the variational integral $I_{F}$ if $I_{F}(u, G) \leqslant I_{F}(w, G)$ for all $w \in C(\bar{G}) \cap W_{p}^{1}(G)$ with $w=v$ in $\partial G$. If $G$ is an open (not necessarily bounded) set in $R^{n}$, then an $A C L^{p}$-function $u$ is called $a$ (free) $F$-extremal if for all open sets $D \subset \subset G, u \mid D$ is an $F$-extremal in $D$ with boundary values $u \mid \partial D$. Clearly an $F$-extremal with boundary values is also a free $F$-extremal, see 3.21.

3.13. REMARK. In the calculus of variations extremals are not usually defined as above. In 3.21 we take an opportunity to clarify the connections between various definitions in the continuous case.

3.14. Euler equation. There is an one-to-one correspondence between variational kernels $F$ satisfy (a)-(d) and corresponding Euler equations. For this purpose we introduce a class of second order partial differential equations. 
Let $G \subset R^{n}$ be open and let $A: G \times R^{n} \rightarrow R^{n}$ be a mapping such that the function $x \mapsto A(x, h(x))$ is measurable whenever $h: G \rightarrow R^{n}$ is measurable and there are numbers $1<p<\infty$ and $\gamma<\infty$ such that for a.e. $x \in G$

$$
|A(x, h)| \leqslant \gamma|h|^{p-1}
$$

for all $h \in R^{n}$. An $A C L^{p}$-function $u: G \rightarrow R$ is called a solution of the equation

$$
\nabla \cdot A(x, \nabla u)=0
$$

in $G$ if

$$
\int_{G} A(x, \nabla u) \cdot \nabla \varphi d m=0
$$

for all $\varphi \in C_{0}^{\infty}(G)$. If $G$ is bounded and $u \in C(\bar{G}) \cap W_{p}^{1}(G)$ is a solution of (3.16) in $G$, then $u$ is said to have boundary values $v \in C(\partial G)$ if $u=v$ in $\partial G$. In this case (3.17) holds, by approximation, for all $\varphi \in W_{p, 0}^{1}(G)$.

We give a proof of the following theorem. The proof is difficult to locate in the literature.

3.18. TheOREM. Suppose that $F$ satisfies (a)-(d). A function $u$ is an F-extremal, free or with boundary values, if and only if it is a corresponding solution of the equation

$$
\nabla \cdot \nabla_{h} F(x, \nabla u)=0 .
$$

Proof. By Remark 3.3 and Lemma 3.5 the mapping $A(x, h)=\nabla_{h} F(x, h)$ satisfies the required assumptions. We may only consider the free case. Let an $A C L^{P_{-}}$ function $u: G \rightarrow R$ be an $F$-extremal and let $\varphi \in C_{0}^{\infty}(G)$. Choose an open set $D \subset \subset G$ with spt $\varphi \subset D$ and set for $t \in R, \psi(t)=I_{F}(u+t \varphi, D)$. Since $u$ is an $F$-extremal $\psi(t) \geqslant \psi(0)$ for all $t$. We show that $\psi$ has a derivative at 0 .

To this end let $|\varepsilon|<1, \varepsilon \neq 0$. Choose $x \in G$ such that $h \mapsto F(x, h)$ has a continuous derivative and (3.2) holds. Write $\Delta F=F\left(x, h_{0}+\varepsilon h\right)-F\left(x, h_{0}\right), h, h_{0}$ $\in R^{n}$. By the mean value theorem $\Delta F / \varepsilon=h \cdot \nabla_{h} F\left(x, h_{0}+\varepsilon^{\prime} h\right)$ and $\varepsilon^{\prime}$ lies between 0 and $\varepsilon$. Lemma 3.5 yields

$$
\begin{aligned}
|\Delta F / \varepsilon| & \leqslant|h|\left|\nabla_{h} F\left(x, h_{0}+\varepsilon^{\prime} h\right)\right| \leqslant \gamma|h|\left|h_{0}+\varepsilon^{\prime} h\right|^{p-1} \\
& \leqslant \gamma 2^{p-1}\left(|h|\left|h_{0}\right|^{p-1}+|h|^{p}\right) .
\end{aligned}
$$

Set $h_{0}=\nabla u$ and $h=\nabla \varphi$. Then for a.e. $x \in D$

$$
|\Delta F / \varepsilon| \leqslant g=\gamma 2^{p-1}\left(|\nabla \varphi||\nabla u|^{p-1}+|\nabla \varphi|^{p}\right)
$$

and Hölder's inequality gives $g \in L^{1}(D)$. On the other hand, for a.e. $x \in D$, $\lim _{\varepsilon \rightarrow 0} \Delta F / \varepsilon=\nabla_{h} F(x, \nabla u) \cdot \nabla \varphi$. Now Lebesgue's dominated convergence theorem implies

$$
\psi^{\prime}(0)=\lim _{\varepsilon \rightarrow 0} \int_{D} \frac{\Delta F}{\varepsilon} d m=\int_{D} \nabla_{h} F(x, \nabla u) \cdot \nabla \varphi d m
$$

and $\psi$ has a derivative at 0 . Since $\psi$ also has a minimum at $0, \psi^{\prime}(0)=0$ and (3.20) gives the required result. 
To prove the other half of the theorem suppose that $u$ is a solution of (3.19). Let $D \subset \subset G$ be open. Since for a.e. $x \in G$ the function $h \mapsto F(x, h)$ is convex and continuously differentiable, a.e. in $D$

$$
F\left(x, h_{1}\right)-F\left(x, h_{2}\right) \geqslant \nabla_{h} F\left(x, h_{2}\right) \cdot\left(h_{1}-h_{2}\right)
$$

for all $h_{1}, h_{2} \in R^{n}$. If $w \in C(\bar{D}) \cap W_{p}^{1}(D)$ and $w=u$ in $\partial D$, then $\varphi=w-u \in$ $W_{p, 0}^{1}(D)$, see e.g. [M3, Lemma 2.2], and a.e. in $D, F(x, \nabla u+\nabla \varphi)-F(x, \nabla u) \geqslant$ $\nabla_{h} F(x, \nabla u) \cdot \nabla \varphi$. By integration

$$
I_{F}(u+\varphi, D)-I_{F}(u, D) \geqslant \int_{D} \nabla_{h} F(x, \nabla u) \cdot \nabla \varphi d m=0
$$

since $u$ is a solution of (3.19) and $\varphi \in W_{p, 0}^{1}(D)$. Thus $I_{F}(w, D)=I_{F}(u+\varphi, D) \geqslant$ $I_{F}(u, D)$ and $u$ is an $F$-extremal in $D$ with boundary values $u \mid \partial D$. This completes the proof.

3.21. REMARK. In the calculus of variations extremals are usually defined with respect to the space $W_{p}^{\prime}(G)$. Suppose that $F: G \times R^{n} \rightarrow R$ satisfies (a)-(d) and $v \in W_{p}^{1}(G)$. A function $u \in W_{p}^{1}(G)$ is called an $F$-extremal in the $W_{p}^{1}$-sense with boundary values $v$ if $u-v \in W_{p, 0}^{1}(G)$ and $I_{F}(u, G) \leqslant I_{F}(w, G)$ for all $w \in W_{p}^{1}(G)$ such that $w-v \in W_{p, 0}^{1}(G)$. A function $u \in \operatorname{loc} W_{p}^{1}(G)$ is called a (free) $F$-extremal in the $W_{p}^{1}$-sense in $G$ if for all open sets $D \subset \subset G, u \mid D$ is an $F$-extremal in the $W_{p}^{1}$-sense with boundary values $u \mid D$ in $D$. It is well known that $F$-extremals in the $W_{p}^{1}$-sense are actually continuous, see e.g. [S, or $\mathbf{M o r}$, and thus $A C L^{p^{-} \text {-functions. }}$ Here we show that an $A C L^{p}$-function $u$ is a free $F$-extremal if and only if it is free extremal in the $W_{p}^{1}$-sense.

To this end suppose that an $A C L^{p}$-function $u$ is a free $F$-extremal in the $W_{p}^{1}$-sense in $G$. Let $D \subset \subset G$ and $w \in C(\bar{D}) \cap W_{p}^{1}(D)$ with $w=u$ in $\partial D$. Then $w-u \in$ $W_{p, 0}^{1}(D)$, see [M3, Lemma 2.2], and thus $I_{F}(u, D) \leqslant I_{F}(w, D)$. Hence $u$ is a free $F$-extremal. For the converse, let $u$ be a free $F$-extremal in $G$ and $D \subset \subset G$ an open set. Pick $w \in W_{p}^{1}(D)$ with $w-u \in W_{p, 0}^{1}(D)$. Choose a sequence of functions $\varphi_{i} \in C_{0}^{\infty}(D)$ with $\varphi_{i} \rightarrow w-u$ in $W_{p}^{1}(D)$. Then $\varphi_{i}+u \in C(\bar{D}) \cap W_{p}^{1}(D)$ and $\varphi_{i}+u$ $=u$ in $\partial D$. Thus $I_{F}(u, D) \leqslant I_{F}\left(u+\varphi_{i}, D\right)$ and it remains to show $I_{F}\left(u+\varphi_{i}, D\right) \rightarrow$ $I_{F}(w, D)$ as $i \rightarrow \infty$. For this note that the mean value theorem and Lemma 3.5 give $C<\infty$ such that a.e. in $D$

$$
\begin{aligned}
& \left|F\left(x, \nabla\left(u+\varphi_{i}\right)\right)-F(x, \nabla w)\right| \\
& \quad \leqslant C\left|\nabla\left(u+\varphi_{i}-w\right)\right|\left(\left|\nabla\left(u+\varphi_{i}\right)\right|^{p-1}+|\nabla w|^{p-1}\right) .
\end{aligned}
$$

Since $\left\|\nabla\left(u+\varphi_{i}-w\right)\right\|_{p} \rightarrow 0$ as $i \rightarrow \infty$, Hölder's inequality gives the required result.

The above reasoning also shows, if $G$ is bounded, that an $F$-extremal $u \in C(\bar{G}) \cap$ $W_{p}^{1}(G)$ with boundary values $v \in C(\bar{G}) \cap W_{p}^{1}(G)$ is an $F$-extremal in the $W_{p}^{1}$-sense with boundary values $v$.

3.22. Existence of extremals. In 1907, H. Lebesgue observed that a kernel satisfying (a)-(c) allows the use of monotone functions, see 2.8. Lemma 3.23 is essentially due to him [L]. Its proof follows from a more general principle considered in 5.6. 
3.23. Lemma. Let $G$ be a bounded open set and let $F$ satisfy (a)-(c) in $G$. If $u \in C(\bar{G}) \cap W_{p}^{1}(G)$, then there is $v \in C(\bar{G}) \cap W_{p}^{1}(G)$ such that $u=v$ in $\partial G, v$ is monotone in $G$, and $I_{F}(v, G) \leqslant I_{F}(u, G)$.

Next we combine Corollary 2.17, Theorem 3.10, and Lemma 3.23 to construct $F$-extremals. The proof is essentially the same as in [M2, Theorem 5.9] and applies only to the borderline case $p=n$, however, see Remark 3.25(b).

3.24. THEOREM. Suppose that $F$ satisfies (a)-(c) in a regular open set $G \subset R^{n}$ with $p=n$. If $v \in C(\bar{G}) \cap W_{n}^{1}(G)$, then there is an F-extremal $u$ in $G$ with boundary values $v$.

Proof. Let $u_{i}, i=1,2, \ldots$, be a minimizing sequence, i.e. $I_{F}\left(u_{i}, G\right) \rightarrow I_{0}=$ $\inf \left\{I_{F}(w, G): w \in \mathscr{F}_{v}\right\}$ where $\mathscr{F}_{v}=\left\{w \in C(\bar{G}) \cap W_{n}^{1}(G): w=v\right.$ in $\left.\partial G\right\}$. By (c) we may assume that $\left\|\nabla u_{i}\right\|_{n} \leqslant M<\infty$ for all $i$. By Lemma 3.23 the sequence $u_{i}$ can be replaced by a minimizing sequence $w_{i} \in \mathscr{F}_{v}$ such that $\left\|\nabla w_{i}\right\|_{n} \leqslant M$ and each $w_{i}$ is monotone. By Corollary 2.17 the family $\left\{w_{i}\right\}$ is equicontinuous and since it is uniformly bounded by $\sup _{\partial G}|v|$, Ascoli's theorem gives a subsequence, denoted again by $w_{i}$, which converges uniformly on $\bar{G}$ to a function $u \in C(\bar{G})$. On the other hand there is a subsequence of $w_{i}$, denoted again by $w_{i}$, such that $\nabla w_{i}$ converges weakly in $L^{n}(G)$ and its limit is the distributional gradient of $u$. Thus $u \in W_{n}^{1}(G)$. By Theorem 3.10

$$
I_{F}(u, G) \leqslant \lim _{i \rightarrow \infty} I_{F}\left(w_{i}, G\right)=I_{0} .
$$

Since $u \in \mathscr{F}_{v}$, this completes the proof.

3.25. REMARKS. (a) In [M2] it was shown that the topological regularity condition on $\partial G$ in 3.24 can be replaced by a weaker condition $M(x, C G)=\infty$ for all $x \in \partial G$. Note that, see [MS], the condition $M(x, C G)=\infty$ is equivalent with the condition

$$
\int_{0} \frac{\varphi(t)}{t} d t=\infty
$$

where $\varphi(t)=\operatorname{cap}_{n}\left(B^{n}(x, t), C G \cap\left(\bar{B}^{n}(x, t / 2) \backslash B^{n}(x, t / 4)\right)\right)$. The condition (3.26) requires more $C G$ than the Wiener criterion

$$
\int_{0} \frac{\psi(t)^{1 /(n-1)}}{t} d t=\infty
$$

$\psi(t)=\operatorname{cap}_{n}\left(B^{n}(x, t), C G \cap \bar{B}^{n}(x, t / 2)\right)$. The Wiener criterion (3.27) is in a natural way connected with the continuous boundary values for solutions of the equation $\nabla \cdot \nabla_{h} F(x, \nabla u)=0$, see [Maz, GZ].

Observe, however, that the use of (3.27) requires some additional assumptions on $F$. On the other hand, for $p=n$ the condition (3.26) is weaker than the usual capacity density condition on $C G$, see [MS].

(b) The reasoning in Theorem 3.24 can also be used for $p>n$, but in this case all the results become easy to obtain by other methods. Note that the method fails for $p<n$ because of Corollary 2.17. 
(c) For all $p>1$ it is easy, by using Sobolev space methods, to prove the existence of an $F$-extremal in the $W_{p}^{1}$-sense, see Remark 3.21. However, we are mainly interested in continuous extremals up to the boundary since they play a central role in the theory of subextremals.

3.28. Extremality as a local property. Suppose that $F$ satisfies (a)-(d) and let $u$ be $A C L^{p}$ in $G$. Then $u$ is an $F$-extremal in $G$ if and only if each point $x \in G$ has a neighborhood $U \subset G$ such that $u \mid U$ is an $F$-extremal in $U$. In other words, the $F$-extremality is a local property. This follows from Theorem 3.18 since by the partition of unity the same question for solutions of $\nabla \cdot \nabla_{h} F(x, \nabla u)=0$ is trivial.

4. Properties of $F$-extremals. Hölder-continuity of solutions of the equation $\nabla \cdot A(x, \nabla u)=0$ has been extensively studied, see e.g. [Mor, S]. In the borderline case $p=n$ we employ a new method which gives a good estimate for the Hölderexponent of $F$-extremals under minimal assumptions on $F$. The same method also gives Harnack's inequality. We close the section with some uniqueness problems and convergence of extremals.

4.1. Hölder-continuity. Let $G$ be an open set in $R^{n}$ and suppose that $F: G \times R^{n} \rightarrow R$ satisfies the assumptions (a)-(c) of $\S 3$ for $p=n$ in $G$. With obvious changes the following lemma remains true for all $p>1$.

4.2. Lemma (STANDARD estimate). Suppose that $u$ is an F-extremal in $G$. If $(D, C)$ is a condenser in $G$, then

$$
\int_{C}|\nabla u|^{n} d m \leqslant n^{n}(\beta(F) / \alpha(F)) \omega(u, D)^{n} \operatorname{cap}_{n}(D, C) .
$$

Proof. Let $\varphi, 0 \leqslant \varphi \leqslant 1$, be admissible for the condenser $E=(D, C)$, i.e. $\varphi \in W_{0}(E)$, see 2.3. Choose an open set $D^{\prime}$ such that spt $\varphi \subset D^{\prime} \subset \subset D$. The function $v=u-\varphi^{n} u$ belongs to $W_{n}^{1}\left(D^{\prime}\right) \cap C\left(\bar{D}^{\prime}\right)$ and $v=u$ on $\partial D^{\prime}$. Set $\beta=\beta(F)$. Since $\nabla v=\left(1-\varphi^{n}\right) \nabla u-n \varphi^{n-1} u \nabla \varphi$, the assumptions (b) and (c) give

$$
F(x, \nabla v(x)) \leqslant\left(1-\varphi(x)^{n}\right) F(x, \nabla u(x))+\beta n^{n}|u(x)|^{n}|\nabla \varphi(x)|^{n}
$$

for a.e. $x \in G$. As $u$ gives the minimum, the inequality (4.3) yields

$$
I_{F}\left(u, D^{\prime}\right) \leqslant I_{F}\left(v, D^{\prime}\right) \leqslant \int_{D^{\prime}}\left(1-\varphi^{n}\right) F(x, \nabla u) d m+\beta n^{n} \int_{D^{\prime}}|u|^{n}|\nabla \varphi|^{n} d m
$$

and since $\varphi \mid C=1$, we have

$$
I_{F}(u, C) \leqslant \beta n^{n} \int_{D^{\prime}}|u|^{n}|\nabla \varphi|^{n} d m \leqslant \beta n^{n} \int_{D}|u|^{n}|\nabla \varphi|^{n} d m .
$$

To prove Lemma 4.2 we may assume $\omega(u, D)<\infty$. If $u$ is an $F$-extremal, $u-\inf _{D} u$ is also, and since $\left|u-\inf _{D} u\right|^{n} \leqslant \omega(u, D)^{n}$ in $D$, the inequality (4.4) implies

$$
I_{F}(u, C) \leqslant \beta n^{n} \omega(u, D)^{n} \int_{D}|\nabla \varphi|^{n} d m .
$$

Taking the infimum over all admissible $\varphi$ for the condenser $(D, C)$, the assumption (c) and (4.5) give the inequality of Lemma 4.2. 
4.6. Remark. If $u$ is an $F$-extremal in $G$, then $u$ is monotone. For if this is not the case, then $u$ can be replaced by a constant function in some subdomain $D \subset \subset G$ and since $u$ is not constant in $D$,

$$
I_{F}(u, D) \geqslant \alpha(F) \int_{D}|\nabla u|^{n} d m>0 .
$$

Hence $u$ cannot give a minimum in $D$.

A combination of Lemmas 4.2 and 2.7 yields the Hölder-continuity of $F$ extremals.

4.7. ThEOREM. Suppose that $u$ is an F-extremal in $G$. If $B^{n}\left(x_{0}, r\right) \subset B^{n}\left(x_{0}, R\right) \subset G$, then

$$
\omega\left(u, B^{n}\left(x_{0}, r\right)\right) \leqslant e(r / R)^{\kappa} \omega\left(u, B^{n}\left(x_{0}, R\right)\right) .
$$

Here $e$ is Neper's number, $\kappa=c_{n}(\alpha(F) / \beta(F))^{1 / n}$ and $c_{n}$ depends only on $n$.

Proof. We may assume $r<R$. For $t>0$ set $B(t)=B^{n}\left(x_{0}, t\right)$ and $S(t)=$ $S^{n-1}\left(x_{0}, t\right)$. By Remark 4.6, $u$ is monotone and hence for $t \leqslant R$

$$
\omega(u, B(t))=\omega(u, S(t)) .
$$

For $0<r_{1}<r_{2} \leqslant R$ set $\rho=\left(r_{1} r_{2}\right)^{1 / 2}$. Then $r_{1}<\rho<r_{2}$ and Lemma 2.7 yields

$$
\omega\left(u, B\left(r_{1}\right)\right)^{n} \log \frac{\rho}{r_{1}} \leqslant A_{n} \int_{B(\rho)}|\nabla u|^{n} d m .
$$

Lemma 4.2 applied to the condenser $E=\left(B\left(r_{2}\right), \bar{B}(\rho)\right)$ implies together with (4.8)

$$
\begin{aligned}
\omega\left(u, B\left(r_{1}\right)\right)^{n} & \leqslant c(\beta(F) / \alpha(F)) \operatorname{cap}_{n} E\left(\log \left(\rho / r_{1}\right)\right)^{-1} \omega\left(u, B\left(r_{2}\right)\right)^{n} \\
& =c^{\prime}(\beta(F) / \alpha(F))\left(\log \left(r_{2} / r_{1}\right)\right)^{-n} \omega\left(u, B\left(r_{2}\right)\right)^{n}
\end{aligned}
$$

and $c$ and $c^{\prime}$ depend only on $n$.

To complete the proof we iterate (4.9). Set $\kappa=\left(c^{\prime} \beta(F) / \alpha(F)\right)^{1 / n}$ and $\lambda=e^{e \kappa}$. If $R>\lambda r$, choose an integer $m \geqslant 1$ such that $\lambda^{m} r<R \leqslant \lambda^{m+1} r$. An iteration of (4.9) using the radii $r, \lambda r, \lambda^{2} r, \ldots, \lambda^{m} r$ gives

$$
\begin{aligned}
\omega(u, B(r)) & \leqslant(\kappa / \log \lambda)^{m} \omega\left(u, B\left(\lambda^{m} r\right)\right)=\left(r / \lambda^{m} r\right)^{1 / \log \lambda} \omega\left(u, B\left(\lambda^{m} r\right)\right) \\
& \leqslant \lambda^{1 / e \kappa}(r / R)^{1 / e \kappa} \omega(u, B(R))=e(r / R)^{1 / e \kappa} \omega(u, B(R)) .
\end{aligned}
$$

If $r<R \leqslant \lambda r$, then $e(r / R)^{1 / e \kappa} \geqslant e(1 / \lambda)^{1 / e \kappa}=1$, and the above estimate is trivial. This completes the proof.

4.10. CoRollary. Suppose that $u: R^{n} \rightarrow R$ is an F-extremal and $F$ satisfies (a)-(c) in $R^{n}$ for $p=n$. If $\kappa$ is the constant of Theorem 4.7 and

$$
\lim _{x \rightarrow \infty} \frac{|u(x)|}{|x|^{\kappa}}=0,
$$

then $u$ is a constant. 
4.11. Corollary (Liouville's TheOrem). Let $u$ and $F$ be as in 4.10. If $u$ is bounded, then $u$ is a constant.

4.12. REMARK. Liouville's theorem is usually proved via Harnack's inequality, see [R3, p. 661; Gr].

4.13. RemarK. It can be proved that every $F$-extremal $u$ where $F$ satisfies (a), (b'), (c) and (d) for $p=n$ belongs to $\operatorname{loc} W_{n+\varepsilon}^{1}(G)$ and $\varepsilon>0$ depends only on $n$ and $\beta(F) / \alpha(F)$, see [ME]. Sobolev's imbedding theorem then gives the Hölder-continuity of $u$. However, the proof for the $n+\varepsilon$-integrability result requires more advanced techniques than those applied here.

4.14. Harnack's inequality. Harnack's inequality for general second order quasilinear elliptic equations is well known, see e.g. [S]. For $F$-extremals we present a simple proof in the borderline case $p=n$. We assume that $F$ satisfies (a)-(c) in $G$ for $p=n$. This proof is based on [Gr]. For our purposes the following form is adequate.

4.15. TheOREM. Let $u$ be a nonnegative F-extremal in $G$. If $B^{n}\left(x_{0}, R\right) \subset G$, then for all $0<r<R$

$$
\sup _{B^{n}\left(x_{0}, r\right)} u \leqslant e^{c(\log (R / r))^{-1}} \inf _{B^{n}\left(x_{0}, r\right)} u .
$$

The constant $c$ depends only on $n$ and $\beta(F) / \alpha(F)$.

For the proof an auxiliary result is needed. Let $u$ be as in 4.15 and, in addition, positive. Set $v=\log u$.

4.16. LEMMA. There is a constant $c_{0}$ depending only on $n$ and $\beta(F) / \alpha(F)$ such that $\int_{C}|\nabla v|^{n} d m \leqslant c_{0} \operatorname{cap}_{n} E$ for each condenser $E=(D, C)$ in $G$.

Proof. We may assume that $D \subset \subset G$ and that $u \geqslant(n-1)^{1 / n}$ in $D$ since otherwise consider $\lambda u$ for large $\lambda$. Observe that $\lambda u$ is an $F_{1}$-extremal for the variational kernel $F_{1}(x, h)=\lambda^{n} F(x, h / \lambda)$ with the same structure constants as $F$. Let $\varphi, 0 \leqslant \varphi \leqslant 1$, be admissible for $E$. Set $h=u+\varphi^{n} u^{1-n}$. Then $h=u$ on $\partial D$ and

$$
\nabla h=\left(1-(n-1) \varphi^{n} / u^{n}\right) \nabla u+n\left(\varphi^{n-1} / u^{n-1}\right) \nabla \varphi .
$$

By convexity and (c) a.e. in $D$

$$
F(x, \nabla h) \leqslant\left(1-(n-1) \varphi^{n} / u^{n}\right) F(x, \nabla u)+\beta(F) n^{n}(n-1)^{1-n}|\nabla \varphi|^{n}
$$

and since $u$ is minimizing in $D$

$$
\begin{aligned}
I_{F}(u, D) \leqslant & I_{F}(h, D) \leqslant I_{F}(u, D)-(n-1) \int_{D}(\varphi / u)^{n} F(x, \nabla u) d m \\
& +\beta(F) n^{n}(n-1)^{1-n} \int_{D}|\nabla \varphi|^{n} d m .
\end{aligned}
$$

This yields

$$
(n-1) \int_{D}(\varphi / u)^{n} F(x, \nabla u) d m \leqslant \beta(F) n^{n}(n-1)^{1-n} \int_{D}|\nabla \varphi|^{n} d m .
$$


The lower bound in (c) gives together with the fact $\varphi \mid C=1$

$$
\begin{aligned}
(n-1) \alpha(F) \int_{C}(|\nabla u| / u)^{n} d m & \leqslant(n-1) \int_{D}(\varphi / u)^{n} F(x, \nabla u) d m \\
& \leqslant \beta(F) n^{n}(n-1)^{1-n} \int_{D}|\nabla \varphi|^{n} d m .
\end{aligned}
$$

This yields the required estimate.

Proof of Theorem 4.15. Let $\varepsilon>0$ and set $v=\log (u+\varepsilon)$. Since $u+\varepsilon$ is an $F$-extremal, the function $u+\varepsilon$ is monotone, see Remark 4.6. Thus $v$ is also monotone and we apply Lemma 4.16 to the condenser $E=\left(B^{n}\left(x_{0}, R\right), \bar{B}^{n}\left(x_{0}, \rho\right)\right)$, $\rho=(R r)^{1 / 2}$, in $G$. Write $B(t)=B^{n}\left(x_{0}, t\right), t>0$. Lemma 2.7 yields

$$
\begin{aligned}
\omega(v, B(r))^{n} \log (\rho / r) & \leqslant \int_{r}^{\rho} \omega(v, B(t))^{n} / t d t=\int_{r}^{\rho} \omega(v, \partial B(t))^{n} / t d t \\
& \leqslant A_{n} \int_{B(\rho)}|\nabla v|^{n} d m \leqslant c_{0} A_{n} \operatorname{cap}_{n} E \\
& =c_{0} A_{n} \omega_{n-1}(\log (R / \rho))^{1-n} .
\end{aligned}
$$

This implies

$$
\log \left(\sup _{B(r)} u+\varepsilon\right)-\log \left(\inf _{B(r)} u+\varepsilon\right) \leqslant \lambda=2\left(c_{0} A_{n} \omega_{n-1}\right)^{1 / n}(\log (R / r))^{-1} .
$$

Thus sup $u+\varepsilon \leqslant e^{\lambda}(\inf u+\varepsilon)$ and letting $\varepsilon \rightarrow 0$ gives the result.

4.17. Uniqueness of extremals. If the condition (b) is replaced by $\left(b^{\prime}\right)$, then it is easy to derive the uniqueness of $F$-extremals with given boundary data. In fact, this result follows from the following comparison principle.

4.18. TheOREM. Suppose that $u_{1}$ and $u_{2}$ are $F$-extremals in a bounded open set $G$ and belong to $C(\bar{G})$. If $u_{1} \geqslant u_{2}$ in $\partial G$, then $u_{1} \geqslant u_{2}$ in $G$.

Proof. Suppose that $u_{1}\left(x_{0}\right)<u_{2}\left(x_{0}\right)$ at some point $x_{0} \in G$. If $0<\varepsilon<u_{2}\left(x_{0}\right)-$ $u_{1}\left(x_{0}\right)$, then there is a domain $D \subset \subset G$ such that $x_{0} \in D, v=u_{1}+\varepsilon=u_{2}$ in $\partial D$ and $v<u_{2}$ in $D$. Consider $w=\left(v+u_{2}\right) / 2$. Now $v$ is also an $F$-extremal and hence $I_{F}\left(u_{1}, D\right) \leqslant I_{F}(w, D)$ and $I_{F}(v, D) \leqslant I_{F}(w, D)$. If $E=\left\{x \in D: \quad \nabla u_{2}(x) \neq\right.$ $\nabla v(x)\}$, then $\left(\mathrm{b}^{\prime}\right)$ yields for a.e. $x \in E$

$$
F(x, \nabla w(x))<\left(F\left(x, \nabla u_{2}(x)\right)+F(x, \nabla v(x))\right) / 2
$$

and this holds as equality for a.e. $x \in D \backslash E$. Thus

$$
I_{F}(w, D)<\left(I_{F}\left(u_{2}, D\right)+I_{F}(v, D)\right) / 2 \leqslant I_{F}(w, D),
$$

if $m(E)>0$. This shows $m(E)=0$ and hence $u_{2}-v=$ const. $=0$ in $D$. Thus $u_{2}=u_{1}+\varepsilon$ in $D$ and especially $u_{2}\left(x_{0}\right)-u_{1}\left(x_{0}\right)=\varepsilon$. This contradiction proves the theorem.

4.19. Corollary. Let $u_{1}$ and $u_{2}$ be as in Theorem 4.18. If $u_{1}=u_{2}$ in $\partial G$, then $u_{1}=u_{2}$ in $G$. 
4.20. Convergence of extremals. Using the comparison principle in Theorem 4.18 it is easy to prove that the set of $F$-extremals is closed under uniform convergence. Observe that the same question for solutions of the equation $\nabla \cdot A(x, \nabla u)=0$ offers additional difficulties, see e.g. [Maz]. We assume that $F$ satisfies (a), (b') and (c) in an open set $G$ with $p=n$. The same proof applies to all cases $p>1$ but then the standard estimate 4.2 for these values of $p$ is used and Theorem 3.24 is replaced by a deeper result.

4.21. TheOREM. Let $u_{i}, i=1,2, \ldots$, be a sequence of F-extremals in $G$ and suppose that $u_{i} \rightarrow u_{0}$ uniformly on compact subsets of $G$. Then $u_{0}$ is an F-extremal.

Proof. Let $D \subset \subset G$ be a regular domain. Lemma 4.2 shows that $u_{0} \in C(\bar{D}) \cap$ $W_{n}^{1}(D)$. Let $\varepsilon>0$. By Theorem 3.24 there is an $F$-extremal $v_{0} \in C(\bar{D}) \cap W_{n}^{1}(D)$ with boundary values $u_{0}$ in $\partial D$. The function $v_{0}+\varepsilon$ is also an $F$-extremal. By the uniform convergence $u_{i}<v_{0}+\varepsilon$ in $\partial D$ for $i \geqslant i_{0}$. By Theorem 4.18, $u_{i} \leqslant v_{0}+\varepsilon$ in $D$ and hence $u_{0} \leqslant v_{0}+\varepsilon$. This shows $u_{0} \leqslant v_{0}$. The same reasoning shows that $u_{0} \geqslant v_{0}$ in $D$. Consequently, $u_{0}=v_{0}$ in $D$. Since this holds in each regular domain $D \subset \subset G, u_{0}$ is an $F$-extremal in $G$.

If an increasing family of $F$-extremals is considered, Harnack's principle is obtained.

4.22. TheOREM. Suppose that $F$ satisfies $(\mathrm{a}),\left(\mathrm{b}^{\prime}\right)$ and $(\mathrm{c})$ in a domain $G$ of $R^{n}$ with $p=n$. If $u_{i}$ is an increasing sequence of $F$-extremals in $G$, then either

(i) $u_{i} \rightarrow u_{0}$ uniformly on compact subsets of $G$, and $u_{0}$ is an F-extremal in $G$, or

(ii) $u_{i}(x) \rightarrow \infty$ at each point $x \in G$.

Proof. Suppose that $\lim u_{i}\left(x_{0}\right)<\infty$ for some $x_{0} \in G$. Since on a compact subset $E$ of $G$ the sequence $u_{i}$ is bounded from below by $m=\inf _{E} u_{1}$ and $u_{i}-m$ is again an $F$-extremal, Harnack's inequality 4.15 and an obvious covering of $E \cup\left\{x_{0}\right\}$ show that $u_{i} \leqslant M<\infty$ in $E$. From this it follows that for each $x \in G$ and $0<R<$ $\operatorname{dist}(x, \partial G)$ there is $M^{\prime}<\infty$ such that $\omega\left(u_{i}, B^{n}(x, R)\right) \leqslant M^{\prime}$ for all $i$. Theorem 4.7 shows the equicontinuity of the family $\left\{u_{i}\right\}$ at each $x \in G$. Since $u_{i}$ is increasing, the convergence must be uniform on compact subsets of $G$. Theorem 4.21 now gives the result.

5. Sub- $F$-extremals. An upper semicontinuous function $u$ : $G \rightarrow R \cup\{-\infty\}$ in a plane open set $G$ is called subharmonic if for all domains $D \subset \subset G$ and all harmonic functions $h \in C(\bar{D})$ in $D$ the inequality $h \geqslant u$ in $\partial D$ implies $h \geqslant u$ in $D$. A subharmonic function is called regular if $u \in C^{2}(G)$. It is well known that $u$ is a regular subharmonic function in $G$ if and only if $\Delta u \geqslant 0$ in $G$. This section is devoted to the corresponding functions in plane and in space when the classical plane Dirichlet kernel $F(x, h)=|h|^{2}$ is replaced by a more general kernel $F(x, h)$ in the borderline case $p=n$.

In comparison problems the uniqueness of an extremal with given boundary data plays an essential role. Hence in the main result of this section, Theorem 5.17, we shall use the condition $\left(b^{\prime}\right)$. It turns out that regular sub- $F$-extremals have a natural interpretation as $A C L^{n}$ functions which obey the comparison principle. 
We also study an obstacle problem in the calculus of variations. The method based on monotone functions, due to $\mathrm{H}$. Lebesgue, has a certain extension even to this situation and yields a relatively easy existence proof in our case $p=n$.

5.1. Definition. Suppose that $F$ satisfies (a)-(c) in an open set $G \subset R^{n}$. An upper semicontinuous function $u: G \rightarrow R \cup\{-\infty\}$ is called a sub-F-extremal in $G$ if for all domains $D \subset \subset G$ and all functions $h \in C(\bar{D})$ such that $h$ is an $F$-extremal in $D$ $h \geqslant u$ on $\partial D$ implies $h \geqslant u$ in $D$.

The following lemma is obvious.

5.2. Lemma. Suppose that $u$ and $v$ are sub-F-extremals in $G$. Then $\max (u, v)$ and $u+\lambda, \lambda \in R$, are sub-F-extremals in $G$. If, in addition, $F$ satisfies $(\mathrm{e})$, then $\lambda u, \lambda \geqslant 0$, is a sub-F-extremal in $G$.

In order to check the comparison condition of Definition 5.1 it suffices to look at regular subdomains of $G$ and rather regular functions $h$.

5.3. Lemma. Let $u: G \rightarrow R \cup\{-\infty\}$ be upper semicontinuous. Then $u$ is a sub-Fextremal in $G$ if and only if for all regular domains $D \subset \subset G$ and all functions $h \in C(\bar{D}) \cap W_{p}^{1}(D)$ which are F-extremals in $D$ the condition $h \geqslant u$ in $\partial D$ implies $h \geqslant u$ in $D$.

Proof. The above condition is clearly necessary. To show the converse let $D \subset \subset G$ be a domain and $h \in C(\bar{D})$ an $F$-extremal in $D$ with $h \geqslant u$ in $\partial D$. Let $x_{0} \in D$ and $\varepsilon>0$. Since $u$ is upper semicontinuous the set $A=\{x \in \bar{D}: h(x)+\varepsilon>$ $u(x)\}$ is open in $\bar{D}$ and contains $\partial D$. Thus there is a regular domain $D^{\prime} \subset \subset D$ such that $\partial D^{\prime} \subset A$ and $x_{0} \in D^{\prime}$. Now $h \in C\left(\bar{D}^{\prime}\right) \cap W_{p}^{1}\left(D^{\prime}\right)$ and since $h+\varepsilon$ is an $F$-extremal in $D^{\prime}$, the condition of Lemma 5.3 gives $h+\varepsilon \geqslant u$ in $D^{\prime}$. Hence $h\left(x_{0}\right)+\varepsilon \geqslant u\left(x_{0}\right)$ and since $\varepsilon>0$ and $x_{0} \in D$ were arbitrary, we have proved $h \geqslant u$ in $D$.

5.4. Definition. Suppose that $F$ satisfies (a)-(c) in an open set $G \subset R^{n}$. A sub-F-extremal $u: G \rightarrow R$ is called a regular sub- $F$-extremal if $u$ is $A C L^{p}$, i.e. $u \in C(G) \cap \operatorname{loc} W_{p}^{1}(G)$.

5.5. Remark. Since $A C L^{p}$-functions form a lattice, Lemma 5.2 is also true for regular sub- $F$-extremals.

Super- $F$-extremals and regular super- $F$-extremals are defined in a similar way. Thus a lower semicontinuous function $u: G \rightarrow R \cup\{\infty\}$ is called a super- $F$-extremal if for all domains $D \subset \subset G$ and all $F$-extremals $h \in C(\bar{D})$ in $D$ the condition $h \leqslant u$ in $\partial D$ implies $h \leqslant u$ in $D$. It is clear that Lemmas 5.2 and 5.3 remain, with obvious modifications, true for super- $F$-extremals.

Next we characterize regular sub- $F$-extremals using variational and differential inequalities in the case $p=n$. To do this we solve a variational problem with an obstacle.

5.6. A modification process with an obstacle. Let $G \subset R^{n}$ be a bounded open set and $u, v \in C(\bar{G})$. For $\alpha \in R$ we define two functions $T_{v}^{+}(u, \alpha)$ and $T_{v}^{-}(u, \alpha)$ on $\bar{G}$ as follows: $T_{v}^{+}(u, \alpha)(x)=\alpha$ if there is a domain $D \subset G$ such that $x \in D, u(y)>\alpha$ $\geqslant v(y)$ for all $y \in D$ and $u(y)=\alpha$ for $y \in \partial D$. In other points $x \in \bar{G}$ set 
$T_{v}^{+}(u, \alpha)(x)=u(x)$. The function $T_{v}^{-}(u, \alpha)$ is defined similarly. If there is a domain $D \subset G$ such that $x \in D, u(y)<\alpha \leqslant v(y)$ for all $y \in D$ and $u(y)=\alpha$ for $y \in \partial D$, then $T_{v}^{-}(u, \alpha)(x)=\alpha$ and $T_{v}^{-}(u, \alpha)(x)=u(x)$ otherwise. Observe that if $\sup _{G} v \leqslant \inf _{G} u$, the function $T_{v}^{+}(u, \alpha)$ does not depend on $v$ and we write $T^{+}(u, \alpha)$ $=T_{v}^{+}(u, \alpha)$. Similarly, if $\inf _{G} v \geqslant \sup _{G} u$, we write $T^{-}(u, \alpha)=T_{v}^{-}(u, \alpha)$. We shall only employ the functions $T^{+}(u, \alpha)$ and $T_{v}^{-}(u, \alpha)$.

For each domain $D \subset G$ we set

$$
\mu^{-}(u, D)=\min _{\partial D} u-\min _{\bar{D}} u, \mu^{+}(u, D)=\max _{\bar{D}} u-\max _{\partial D} u .
$$

It is easy to see that the following lemma is true, cf. [Mor, Lemma 4.3.2].

5.7. LemMA. (a) $T^{+}(u, \alpha) \leqslant u$ in $\bar{G}$ and $T^{+}(u, \alpha)=u$ in $\partial G$.

(b) For each continuum $S \subset \bar{G}$

$$
\omega\left(T^{+}(u, \alpha), S\right) \leqslant \omega(u, S)
$$

and thus $T^{+}(u, \alpha)$ is continuous on $\bar{G}$.

(c) $\mu^{-}\left(T^{+}(u, \alpha), D\right) \leqslant \mu^{-}(u, D), \mu^{+}\left(T^{+}(u, \alpha), D\right) \leqslant \mu^{+}(u, D)$ whenever $D \subset G$ is a domain.

(d) If $u \in C(\bar{G}) \cap W_{p}^{1}(G)$, then $T^{+}(u, \alpha) \in C(\bar{G}) \cap W_{p}^{1}(G)$ and $I_{F}\left(T^{+}(u, \alpha), G\right)$ $\leqslant I_{F}(u, G)$.

Here the kernel $F$ satisfies (a)-(c) in $G$.

A similar lemma holds for $T^{-}(u, \alpha)$. However, we need a corresponding obvious lemma for $T_{v}^{-}(u, \alpha)$ where $v(x) \geqslant u(x)$ for all $x \in \bar{G}$. Write $w=T_{v}^{-}(u, \alpha)$.

5.8. LEMMA. (a) $w \geqslant u$ in $\bar{G}, w=u$ in $\partial G$, and $v \geqslant w$ in $\bar{G}$.

(b) $\omega(w, S) \leqslant \omega(u, S)$ whenever $S \subset \bar{G}$ is a continuum.

(c) $\mu^{-}(w, D) \leqslant \mu^{-}(u, D), \mu^{+}(w, D) \leqslant \mu^{+}(u, D)$.

(d) If in a domain $D \subset G, \min _{\partial D} w \geqslant \alpha \leqslant \inf _{D} v$, then $\inf _{D} w \geqslant \alpha$.

(e) If $u \in C(\bar{G}) \cap W_{p}^{1}(G)$, then $w \in C(\bar{G}) \cap W_{p}^{1}(G)$ and $I_{F}(w, G) \leqslant I_{F}(u, G)$.

5.9. Lemma. Let $G$ be a bounded open set in $R^{n}$ and let $F$ satisfy (a)-(c) in $G$. Suppose that $u \in C(\bar{G}) \cap W_{p}^{1}(G)$ and $v \in C(\bar{G})$ and that $u \leqslant v$ in $\bar{G}$. Then there is $a$ function $w \in C(\bar{G}) \cap W_{p}^{1}(G)$ such that (i) $w|\partial G=u| \partial G$, (ii) $w \leqslant v$ in $\bar{G}$, (iii) $I_{F}(w, G) \leqslant I_{F}(u, G)$, and (iv) if $D \subset G$ is a domain, then

$$
\omega(w, D) \leqslant \max (\omega(w, \partial D), \omega(v, D)) .
$$

5.10. RemarK. This lemma extends a result of H. Lebesgue [L], cf. Lemma 3.23. It reduces to Lemma 3.23 if the obstacle $v$ has no effect, i.e. $w$ can be chosen to be monotone by taking $v(x)=\sup _{G} u$ for all $x \in G$ and then $\omega(w, D)=\omega(w, \partial D)$ by (iv).

Proof of Lemma 5.9. Let $M=\sup _{G} u$ and $m=\inf _{G} u$. For $i=1,2, \ldots$ set $\alpha_{i j}=M-2^{-i} j(M-m), j=1, \ldots, 2^{i}-1$. Define a family $w_{i j}, i=1,2, \ldots, j=$ $1, \ldots, 2^{i}-1$, of functions in $C(\bar{G}) \cap W_{p}^{1}(G)$ inductively as follows:

$$
\begin{aligned}
& w_{11}=T^{+}\left(u, \alpha_{11}\right), \\
& w_{i 1}=T^{+}\left(w_{i-1,2^{i-1}-1}, \alpha_{i 1}\right), \quad i=2,3, \ldots, \\
& w_{i j}=T^{+}\left(w_{i, j-1}, \alpha_{i j}\right), \quad i=2,3, \ldots, 2 \leqslant j \leqslant 2^{i}-1 .
\end{aligned}
$$


Set $f_{i}=w_{i 1}$. Lemma 5.7 gives the following properties of the functions $f_{i}$.

(I) $u \geqslant f_{1} \geqslant f_{2} \geqslant \cdots$ in $\bar{G}, f_{i}=u$ in $\partial G$.

(II) The family $\left\{f_{i}\right\}$ is equicontinuous and bounded.

(III) $f_{i} \in C(\bar{G}) \cap W_{p}^{1}(G)$.

(IV) $I_{F}\left(f_{i}, G\right) \leqslant I_{F}(u, G)$ and by the assumption (c) of 3.1 the sequence $\left\|\nabla f_{i}\right\|_{p}$ is bounded.

Let $f$ be the uniform limit of the sequence $\left(f_{i}\right)$. It is not difficult to see that by the construction

$$
\mu^{+}\left(f_{i}, D\right) \leqslant 2^{-i+1}(M-m)
$$

for every domain $D \subset G$. Thus $f \in C(\bar{G}) \cap W_{p}^{1}(G), f \leqslant v$ in $\bar{G}, f=u$ in $\partial G$, $\mu^{+}(f, D)=0$ for every domain $D \subset G$, and by the semicontinuity Theorem 3.10, $I_{F}(f, G) \leqslant I_{F}(u, G)$.

Next we employ the same construction but using the function $T_{v}^{-}$and starting with $f$. For $i=1,2, \ldots$ and $j=1, \ldots, 2^{i}-1$ we define new numbers $\alpha_{i j}$ and functions $w_{i j}$ inductively as follows:

$$
\begin{aligned}
\alpha_{i j} & =m+2^{-i j}(M-m), \\
w_{11} & =T_{v}^{-}\left(f, \alpha_{11}\right), \\
w_{i 1} & =T_{v}^{-}\left(w_{i-1,2^{i-1}-1}, \alpha_{i 1}\right), \quad i=2,3, \ldots, \\
w_{i j} & =T_{v}^{-}\left(w_{i, j-1}, \alpha_{i j}\right), \quad i=2,3, \ldots, j=2, \ldots, 2^{i}-1 .
\end{aligned}
$$

Set again $f_{i}=w_{i 1}, i=1,2, \ldots$ Then Lemma 5.8 and (5.11) yield:

(I) $f \leqslant f_{1} \leqslant f_{2} \leqslant \cdots \leqslant v$ in $\bar{G}, f_{i}=u$ in $\partial G$,

(II) the family $f_{i}$ is equicontinuous,

(III) $f_{i} \in C(\bar{G}) \cap W_{p}^{\mathrm{l}}(G), I_{F}\left(f_{i}, G\right) \leqslant I_{F}(u, G)$ and hence the sequence $\left\|\nabla f_{i}\right\|_{p}$ is bounded, and

(IV) $\mu^{+}\left(f_{i}, D\right)=0$ for all domains $D \subset G$.

By construction it is easy to see, using Lemma 5.8(d), that

$$
\inf _{D} f_{i} \geqslant \min \left(\inf _{\partial D} f_{i}, \inf _{D} v\right)-2^{-i+1}(M-m)
$$

for each domain $D \subset G$. Let $w$ be the uniform limit of the sequence $f_{i}$. Now $w$ clearly satisfies the properties (i)-(iii) and it remains to verify (iv).

To this end let $D \subset G$ be a domain. Then (IV) and (5.12) yield

$$
\omega(w, D)=\sup _{D} w-\inf _{D} w \leqslant \sup _{\partial D} w-\min \left(\inf _{\partial D} w, \inf _{D} v\right) .
$$

If $c=\min \left(\inf _{\partial D} w, \inf _{D} v\right)=\inf _{\partial D} w$, we have proved (iv). If $c=\inf _{D} v$, then since $w \leqslant v$,

$$
\omega(w, D) \leqslant \sup _{\partial D} w-\inf _{D} v \leqslant \sup _{D} v-\inf _{D} v=\omega(v, D) .
$$

Thus (iv) again follows. This completes the proof.

5.13. REMARK. Lemma 5.9 can be used in obstacle problems in a similar fashion as monotone functions in free variational problems. The next subsection contains an application of this type. 
5.14. Variational problem with an obstacle. Suppose that $F: G \times R^{n} \rightarrow R$ satisfies (a)-(c) in a regular bounded open set $G$ of $R^{n}$ with $p=n$. Let $v, h \in C(\bar{G})$ and suppose that the set

$$
\mathscr{F}(v, h)=\left\{w \in C(\bar{G}) \cap W_{n}^{1}(G): w \leqslant v \text { in } \bar{G}, w=h \text { in } \partial G\right\}
$$

is nonempty.

5.15. Theorem. There is $u \in \mathscr{F}(v, h)$ such that $I_{F}(u, G)=\inf \left\{I_{F}(w, G): w \in\right.$ $\mathscr{F}(v, h)\}$.

Proof. The proof is based on Lemmas 5.9 and 2.10. By Lemma 5.9 there is a minimizing sequence $w_{i} \in \mathscr{F}(v, h)$ such that on each domain $D \subset G$

$$
\omega\left(w_{i}, D\right) \leqslant \max \left(\omega\left(w_{i}, \partial D\right), \omega(v, D)\right) .
$$

By Lemma 2.10 the sequence $w_{i}$ is equicontinuous. The rest of the proof is similar to the proof of Theorem 3.24.

5.16. Variational and differential inequalities for regular sub-F-extremals. Assume that $F: G \times R^{n} \rightarrow R$ satisfies (a), (b'), (c) and (d) of 3.1 in an open set $G$ of $R^{n}$ for $p=n$.

5.17. THEOREM. Let $u$ be an $A C L^{n}$-function in $G$. Then the following conditions are equivalent:

(i) $u$ is a regular sub-F-extremal in $G$.

(ii) For all nonnegative $\varphi \in C_{0}^{\infty}(G)$

$$
\int_{\mathrm{spt} \varphi} F(x, \nabla u) d m \leqslant \int_{\mathrm{spt} \varphi} F(x, \nabla(u-\varphi)) d m .
$$

(iii) For all nonnegative $\varphi \in C_{0}^{\infty}(G)$

$$
\int_{G} \nabla_{h} F(x, \nabla u) \cdot \nabla \varphi d m \leqslant 0 .
$$

Proof. We first show (i) $\Rightarrow$ (ii). Let $u$ be a regular sub- $F$-extremal in $G$ and let $D \subset \subset G$ be a regular domain. Write $\mathscr{F}(u)=\left\{v \in C(\bar{D}) \cap W_{n}^{1}(D): v \leqslant u, v=u\right.$ in $\partial D$ \}. By Theorem 5.15 there is $w \in \mathscr{F}(u)$ with

$$
I_{F}(w, D)=\inf \left\{I_{F}(v, D): v \in \mathscr{F}(u)\right\} .
$$

If $I_{F}(u, D) \leqslant I_{F}(w, D)$, then for all nonnegative $\varphi \in C_{0}^{\infty}(D), \quad I_{F}(u, D) \leqslant$ $I_{F}(u-\varphi, D)$ and (5.18) follows. We show that the case $I_{F}(u, D)>I_{F}(w, D)$ is impossible.

To this end let $A$ be a component of the open set $\{x \in D: w(x)<u(x)\}$. Now $w=u$ in $\partial A$. Let $\eta \in C_{0}^{\infty}(A),|\eta| \leqslant 1$, and set $\lambda=\min _{\mathrm{spt} \eta}(u(x)-w(x))>0$. Let $|\varepsilon|<\lambda$. Then $\varepsilon \eta \leqslant u-w$ in $\bar{A}$ and hence $w+\varepsilon \eta \leqslant u$ in $A$. By the definition of $w$, $I_{F}(w, A) \leqslant I_{F}(w+\varepsilon \eta, A)$ for all $|\varepsilon| \leqslant \lambda$. But now we can use the proof of Theorem 3.18 to conclude

$$
\int_{A} \nabla_{h} F(x, \nabla w) \cdot \nabla \eta d m=0
$$


for all $\eta \in C_{0}^{\infty}(A),|\eta| \leqslant 1$. Clearly (5.20) then holds for all $\eta \in C_{0}^{\infty}(A)$. By Theorem 3.18 the function $w$ is an $F$-extremal in $A$. By (i), $w \geqslant u$ in $A$, hence $A=\varnothing$. Consequently, $w \geqslant u$ in $D$ and thus $w=u$ which shows $I_{F}(w, D)=I_{F}(u, D)$ and the second case is impossible.

Next we prove (ii) $\Rightarrow$ (iiii). Let $\varphi \in C_{0}^{\infty}(G)$ be nonnegative and $\varepsilon>0$. By (ii)

$$
0 \geqslant \int_{\text {spt } \varphi} \frac{F(x, \nabla u)-F(x, \nabla(u-\varepsilon \varphi))}{\varepsilon} d m .
$$

Thus, cf. the proof of Theorem 3.18,

$$
\begin{aligned}
\int_{G} \nabla_{h} F(x, \nabla u) \cdot \nabla \varphi d m & =\int_{\operatorname{spt} \varphi} \lim _{\varphi \rightarrow 0} \frac{F(x, \nabla u)-F(x, \nabla(u-\varepsilon \varphi))}{\varepsilon} d m \\
& =\lim _{\varepsilon \rightarrow 0} \int_{\text {spt } \varphi} \frac{F(x, \nabla u)-F(x, \nabla(u-\varepsilon \varphi))}{\varepsilon} d m \leqslant 0 .
\end{aligned}
$$

This shows that (5.19) holds.

To complete the proof it remains to show (iii) $\Rightarrow$ (i). Suppose that (i) is not true. Then there is, by Lemma 5.3, a domain $D \subset \subset G$ and an $F$-extremal $h \in C(\bar{D}) \cap$ $W_{n}^{1}(D)$ such that $h \geqslant u$ in $\partial D$ but $h\left(x_{0}\right)<u\left(x_{0}\right)$ at some point $x_{0} \in D$. Choosing a smaller subdomain of $D$ we may assume that $h=u$ in $\partial D$ and $h<u$ in $D$. Setting $\varphi=u-h$ in $D$ we obtain a function $\varphi \in W_{n, 0}^{1}(D)$ with $\varphi \geqslant 0$. Since (5.19) clearly holds for nonnegative functions in $W_{n, 0}^{1}(G)$, it is true for the function $\varphi$. On the other hand, ( $\left.\mathrm{b}^{\prime}\right)$ and (d) of 3.1 imply for a.e. $x \in G$

$$
F\left(x, h_{2}\right)-F\left(x, h_{1}\right) \leqslant \nabla_{h} F\left(x, h_{2}\right) \cdot\left(h_{2}-h_{1}\right) .
$$

Hence $I_{F}(u, D)-I_{F}(h, D) \leqslant \int_{D} \nabla_{h} F(x, \nabla u) \cdot \nabla \varphi d m \leqslant 0$. This means that $u$ is a minimizing function in $D$ with boundary values $u|\partial D=h| \partial D$. Now Theorem 4.18 yields $u=h$ in $D$ and the case $h<u$ in $D$ is impossible. This completes the proof of the theorem.

5.21. Remark. Let $u$ be an $A C L^{n}$-function in $G$. The following fact can be easily derived from (iii) of Theorem 5.17. The function $u$ is a regular sub- $F$-extremal in $G$ if and only if for every $x \in G$ there is a neighborhood $U$ such that $u \mid U$ is a regular sub- $F$-extremal in $U$. In other words, regular sub- $F$-extremality is a local property.

5.22. Sub-F-extremality as a local property. In the last section we need the sub- $F$ extremality as a local property. This is well known in the classical subharmonic case. The celebrated method of $\mathrm{H}$. A. Schwarz is used to prove a result of this type.

We assume that $G$ is an open set in $R^{n}$ and $F$ satisfies (a), (b'), (c) and (d) for $p=n$ in $G$.

A family $\Re$ of domains $V \subset \subset G$ is called a regular base in $G$ if for all domains $D \subset G$ and compact set $K \subset D$ there is a finite subfamily $V_{1}, \ldots, V_{k}$ in $\mathscr{B}$ such that $K \subset \cup V_{i} \subset D$ and any union of the sets $V_{1}, \ldots, V_{k}$ is a regular open set in the sense of 2.9. The subfamily $\left\{V_{i}\right\}$ is called a regular cover of $K$ in $D$.

Let $u$ : $G \rightarrow[-\infty, \infty)$ be an upper semicontinuous function and $D \subset \subset G$ an open set. We say that $u$ satisfies the $F$-comparison principle in $D$ if for each $F$-extremal $h \in C(\bar{D}) \cap W_{n}^{1}(D)$ in $D$ the condition $h \geqslant u$ in $\partial D$ implies $h \geqslant u$ in $D$. By Lemma 
5.3, $u$ is a sub- $F$-extremal if $u$ satisfies the $F$-comparison principle in all regular domains $D \subset \subset G$. The next lemma still restricts the class of domains $D$ needed.

5.23. LemMa. Let $\mathscr{B}$ be a regular base in $G$. If $u$ satisfies the $F$-comparison principle in each $V \in \mathscr{B}$, then $u$ is a sub-F-extremal.

Proof. By Lemma 5.3 it suffices to show that $u$ satisfies the $F$-comparison principle in each regular domain $D \subset \subset G$. Let $h \in C(\bar{D}) \cap W_{n}^{1}(D)$ be an $F$ extremal in $D$ and $h \geqslant u$ in $\partial D$. Fix $\varepsilon>0$. By the upper semicontinuity of $u$ the set $K=\{x \in D: u(x) \geqslant h(x)+\varepsilon\}$ is compact in $D$. Since $\mathscr{B}$ is a regular base, there is a regular cover $\left\{V_{1}, \ldots, V_{k}\right\} \subset \mathscr{B}$ of $K$ in $D$. Set $V=\cup V_{i}$. If $x \in \partial V$, then $u(x)<$ $h(x)+\varepsilon$ and it remains to show that the same holds in $V$. By induction it suffices to prove the following lemma.

5.24. Lemma. Suppose that $U, V$ and $U \cup V$ are regular domains in $G$. If $u$ satisfies the $F$-comparison principle in $U$ and in $V$, then it satisfies the same principle in $U \cup V$.

Proof. For the proof the method of H. A. Schwarz is used. Set $W=U \cup V$. Let $\varepsilon>0$ and let $h \in C(\bar{W}) \cap W_{n}^{1}(W)$ be an $F$-extremal in $W$ with $h \geqslant u+\varepsilon$ in $\partial W$. Pick $\varphi_{1} \in C(\bar{W}) \cap W_{n}^{1}(W)$ such that $\varphi_{1} \geqslant u$ in $\bar{W}$ and $\varphi_{1}=h$ in $\partial W$. To complete the proof we construct an $F$-extremal $w \in C(\bar{W}) \cap W_{n}^{1}(W)$ with boundary values $h$ and $w \geqslant u$ in $W$. Theorem 4.18 then implies $h=w$ and thus $h \geqslant u$.

Consider the set

$$
\mathscr{F}=\left\{\psi \in C(\bar{W}) \cap W_{n}^{1}(W): \psi=h \text { in } \partial W, \psi \geqslant \varphi_{1} \text { in } \bar{W}\right\} .
$$

Now Theorem 5.15 holds, by symmetry, as well if the obstacle $\varphi_{1}$ restricts the functions $\psi$ from below. Hence there is $\psi_{1} \in \mathcal{F}$ with

$$
I_{F}\left(\psi_{1}, W\right)=\inf \left\{I_{F}(\psi, W): \psi \in \mathscr{F}\right\} .
$$

If now $\varphi \in C_{0}^{\infty}(W), \varphi \geqslant 0$, then $I_{F}\left(\psi_{1}, W\right) \leqslant I_{F}\left(\psi_{1}+\varphi, W\right)$ and by Theorem 5.17 for regular super- $F$-extremals, $\psi_{1}$ is a regular super- $F$-extremal in $W$. Starting from $\psi_{1}$ we construct a sequence of functions $v_{i}, i=0,1,2, \ldots$, as follows. Set $v_{0}=\psi_{1}$. Let $v_{1} \in C(\bar{W}) \cap W_{n}^{1}(W)$ be the $F$-extremal in $U$ with boundary values $v_{0}$ and in $W \backslash U, v_{1}=v_{0}$ and continue by induction: $v_{2 i}$ is the $F$-extremal in $V$ with boundary values $v_{2 i-1}$ and in $W \backslash V, v_{2 i}=v_{2 i-1}, v_{2 i+1}$ is the $F$-extremal with boundary values $v_{2 i}$ in $U$ and $v_{2 i+1}=v_{2 i}$ in $W \backslash U, i=1,2, \ldots$ Then $v_{i} \in C(\bar{W}) \cap W_{n}^{\prime}(W)$ and

$$
I_{F}\left(v_{i}, W\right) \geqslant I_{F}\left(v_{i+1}, W\right) \text {. }
$$

On the other hand, each $v_{i}$ is a super- $F$-extremal and $v_{0} \geqslant v_{1} \geqslant v_{2} \geqslant \cdots \geqslant u$ since the comparison principle holds in $U$ and in $V$. Hence the limit $w=\lim _{i \rightarrow \infty} v_{i}$ exists in $\bar{W}$ and $w \geqslant u$.

Next we show that the sequence $v_{i}$ tends to $w$ uniformly. For this it clearly suffices to show that the sequence $v_{i}$ is equicontinuous in $\bar{W}$. Let $x_{0} \in W$. Since $v_{0} \geqslant v_{1} \geqslant \ldots$ and the sequence $v_{i}$ is bounded and either $v_{i}$ or $v_{i+1}$ is an $F$-extremal in a neighborhood of $x_{0}$, Theorem 4.7 implies the equicontinuity of $v_{i}$ 's at $x_{0}$. If $x_{0} \in \partial W \backslash(\partial U \cap \partial V)$, then each $v_{i}, i \geqslant 2$, is monotone in $W \cap B$, where $B$ is a fixed neighborhood of $x_{0}$ and since $I_{F}\left(v_{i}, W\right) \leqslant M<\infty$, we can use the proof of Lemma 
2.10, see also Corollary 2.17 , to conclude the equicontinuity at $x_{0}$. Finally, let $x_{0} \in \partial W \cap \partial U \cap \partial V$. We may assume $x_{0}=0$. Suppose that the sequence $v_{i}$ is not equicontinuous at 0 . Then there is $\varepsilon>0$ such that for each $r>0$ there is some $v_{i}$, $i \geqslant 2$, with $\omega\left(v_{i}, \bar{W} \cap \bar{B}^{n}(r)\right) \geqslant \varepsilon$. Pick $\rho>0$ such that for $t \in(0, \rho), S^{n-1}(t) \cap \partial W$ $\neq \varnothing$ and $\omega\left(\psi_{1}, \bar{W} \cap \bar{B}^{n}(\rho)\right)<\varepsilon / 8$. For $r \in(0, \rho)$ let $\omega\left(v_{i}, \bar{W} \cap \bar{B}^{n}(r)\right) \geqslant \varepsilon$ and set

$$
u_{i}(x)=\frac{2}{\varepsilon}\left(\psi_{1}(0)-\varepsilon / 4-v_{i}(x)\right) \text {. }
$$

Write $A=\left\{x \in \bar{W} \cap \bar{B}^{n}(\rho): u_{i}(x)>1\right\}$. Then $A \cap B^{n}(r) \neq \varnothing$ and $\bar{A} \subset W$. Now $A$ meets each $S^{n-1}(t), t \in(r, \rho)$, since if $B$ is a component of $A$ in $B^{n}(t)$, then $v_{i}(x)=\psi_{1}(0)-3 \varepsilon / 4$ in $\partial B$ and $v_{i}(x)<\psi_{1}(0)-3 \varepsilon / 4$ in $B$. Since $v_{i}, i \geqslant 2$, is a super- $F$-extremal, it cannot have an essential minimum. Thus $B=\varnothing$.

We can now proceed as in the proof of Lemma 2.10. With suitably chosen spherical caps $C\left(x_{t}, \varphi_{t}\right) \subset S^{n-1}(t) \cap \bar{W}, t \in(r, \rho), \omega\left(u_{i}, C\left(x_{t}, \varphi_{t}\right)\right) \geqslant 1$ and

$$
\begin{aligned}
\log (\rho / r) & \leqslant \int_{r}^{\rho} \omega\left(u_{i}, C\left(x_{t}, \varphi_{t}\right)\right)^{n} / t d t \\
& \leqslant A_{n} \int_{W}\left|\nabla u_{i}\right|^{n} d m=A_{n}(2 / \varepsilon)^{n} \int_{W}\left|\nabla v_{i}\right|^{n} d m \\
& \leqslant A_{n} \alpha(F)^{-1}(2 / \varepsilon)^{n} I_{F}\left(v_{i}, W\right) \leqslant A_{n} \alpha(F)^{-1}(2 / \varepsilon)^{n} M<\infty
\end{aligned}
$$

where Lemma 2.7 has been used. Letting $r \rightarrow 0$ gives a contradiction.

Thus $w \in C(\bar{W})$ and $w=h$ in $\partial W$. Since $I_{F}\left(v_{i}, W\right) \leqslant M, w \in W_{n}^{1}(W)$. Since $v_{i}$ is an $F$-extremal either in $U$ or in $V$, Theorem 4.21 implies that $w$ is an $F$-extremal in $U$ and in $V$. Since the $F$-extremality is a local property, $w$ is an $F$-extremal in $W$. This completes the proof.

6. Quasiregular mappings and variational integrals. This section is devoted to the invariance of variational integrals under quasiconformal and quasiregular mappings.

6.1. Quasiregular mappings and variational kernels. Let $G$ and $G^{\prime}$ be open sets in $R^{n}$ and $f: G \rightarrow G^{\prime}$ a continuous map such that the partial derivatives of the coordinate functions of $f$ exist a.e. in $G$. Suppose that $F: G^{\prime} \times R^{n} \rightarrow R$ satisfies (a)-(c) for $p=n$ in $G^{\prime}$. Define $f^{\neq} F: G \times R^{n} \rightarrow R$ as

$$
f^{\neq} F(x, h)=\left\{\begin{array}{l}
F\left(f(x), J(x, f)^{1 / n} f^{\prime}(x)^{-1^{*}} h\right), \quad \text { if } J(x, f) \neq 0, \\
|h|^{n}, \quad \text { if } J(x, f)=0 \text { or } J(x, f) \text { does not exist. }
\end{array}\right.
$$

By $A^{*}$ we denote the adjoint of a linear mapping $A: R^{n} \rightarrow R^{n}$.

A continuous mapping $f: G \rightarrow G^{\prime}$ is called quasiregular (qr) if $f$ is $A C L^{n}$ and for some $K \geqslant 1$

$$
\left|f^{\prime}(x)\right|^{n} \leqslant K J(x, f)
$$

a.e. in $G$. Here $|A|$ is the sup-norm of a linear map $A$. The smallest $K$ for which (6.2) holds is denoted by $K_{0}(f)$, the outer dilatation of $f$. The inner dilatation $K_{I}(f)$ of $f$ is defined as the smallest for which the inequality

$$
K l\left(f^{\prime}(x)\right)^{n} \geqslant J(x, f)
$$

holds a.e. in $G$. Here $l\left(f^{\prime}(x)\right)=\inf _{|h|=1}\left|f^{\prime}(x) h\right|$. 
For the theory of qr mappings we refer to [MRV1]. For later applications we recall some basic properties of these mappings. If $G \subset R^{n}$ is a domain and $f: G \rightarrow G^{\prime}$ is $\mathrm{qr}$, then $f$ is either a constant or a discrete, open, and sense-preserving mapping [R3]. In the latter case a domain $D \subset \subset G$ is called a normal domain of $f$ if $f \partial D=\partial f D$. Then $f$ defines a closed mapping $D \rightarrow f D$ which is finite-to-one. From [MRV1] it follows that $N(f, D)=\Sigma_{x \in f^{-1}(y) \cap D} i(x, f)$ for all $y \in f D$. Here $N(f, D)$ $=\sup _{y \in R^{n}} \operatorname{card}\left(f^{-1}(y) \cap D\right)<\infty$ and $i(x, f)$ is the local topological index of $f$ at $x$. Note that for each $x \in G$ there exists $r_{0}>0$ such that for $0<r \leqslant r_{0}$ the $x$-component $U(x, f, r)$ of $f^{-1} B^{n}(f(x), r)$ is a normal domain of $f$ with the additional property $U(x, f, r) \cap f^{-1}(f(x))=\{x\}$. Then $U(x, f, r)$ is called a normal neighborhood of $x$. We shall also use the analytic properties of $f$ e.g. if $A \subset G$, then $m(A)=0$ if and only if $m(f A)=0$ and $f$ is a.e. differentiable, see [MRV1].

A mapping $f: G \rightarrow G^{\prime}$ is called quasiconformal (qc) if it is quasiregular and injective. Observe that if $f: G \rightarrow G^{\prime}$ is a qc homeomorphism, then $f^{-1}: G^{\prime} \rightarrow G$ is also a qc map and $K_{0}(f)=K_{I}\left(f^{-1}\right)$ and $K_{I}(f)=K_{0}\left(f^{-1}\right)$, see [V].

The connection between $F$ and $f^{\neq} F$ is given by the following lemma.

6.4. Lemma. Suppose that $f: G \rightarrow G^{\prime}$ is qr and $F: G^{\prime} \times R^{n} \rightarrow R$ satisfies the assumptions (a)-(c) for $p=n$ in $G^{\prime}$. Then $f^{\neq} F$ satisfies the same assumptions in $G$ for $p=n$ and

$$
\alpha\left(f^{\neq} F\right) \geqslant K_{0}(f)^{-1} \alpha(F), \quad \beta\left(f^{\neq} F\right) \leqslant K_{I}(f) \beta(F) .
$$

Moreover, if $F$ satisfies ( $\left.\mathrm{b}^{\prime}\right)$, (d) or (e) in $G^{\prime}$, then $f^{\neq} F$ satisfies the same assumptions in $G$.

Proof. Considering separately the components of $G$ we may assume that $G$ is a domain. If $f$ is a constant map, then $K_{0}(f)=1=K_{I}(f)$, and the result is immediate. Suppose that $f$ is nonconstant. Let $A=\{x \in G: J(x, f)>0$ and $f$ is differentiable at $x$ \}. Since $m(f C)=0$ if and only if $m(C)=0$, see [MRV1, Theorem 8.4], the assumptions (a) and (b) for $f^{\neq} F$ follow easily. For a.e. $x \in A$ and all $h \in R^{n}$

$$
\begin{aligned}
f^{\neq} F(x, h) & =F\left(f(x), J(x, f)^{1 / n} f^{\prime}(x)^{-1^{*}} h\right) \\
& \leqslant \beta(F) J(x, f)\left|f^{\prime}(x)^{-1^{*}} h\right|^{n} \leqslant \beta(F) J(x, f)\left|f^{\prime}(x)^{-1}\right|^{n}|h|^{n}
\end{aligned}
$$

where the assumption (c) for $F$ has been used. On the other hand

$$
\left|f^{\prime}(x)^{-1}\right|^{n} \leqslant K_{I}(f) \operatorname{det}\left(f^{\prime}(x)^{-1}\right)=K_{I}(f) J(x, f)^{-1}
$$

a.e. in $A$. Thus (6.5) yields $f^{\neq} F(x, h) \leqslant K_{I}(f) \beta(F)|h|^{n}$.

Since $m(G \backslash A)=0$, see [MRV, 2.26 and 8.2], this holds a.e. in $G$. The lower bound follows similarly using the inequality $l\left(f^{\prime}(x)^{-1}\right)^{n} \geqslant K_{0}(f)^{-1} \operatorname{det}\left(f^{\prime}(x)^{-1}\right)$ a.e. in $G$. The assumptions ( $\left.\mathrm{b}^{\prime}\right)$, (d), and (e) for $f^{\neq} F$ are immediate. The lemma follows.

6.7. Invariance of variational integrals. If $f: G \rightarrow G^{\prime}$ is a conformal mapping, then $F$ and $f^{\neq} F$ are essentially of the same character whenever $F$ satisfies (a)-(c) for $p=n$ in $G^{\prime}$. If $F$, in addition, satisfies (e), then this invariance property can be expressed in terms of the integrals $I_{F}$ and $I_{f^{\neq} F}$. 
We start with a well-known fact, see [V, p. 113; RR, Theorem 2, p. 363; G, Theorem 6.3]. Suppose that $f: G \rightarrow G^{\prime}$ is a qc homeomorphism and $h: G^{\prime} \rightarrow \dot{R}$ a measurable function. Then for each measurable $A \subset G^{\prime}$

$$
\int_{A} h d m=\int_{f^{-1} A} h(f(x)) J(x, f) d m(x),
$$

if one of the integrals in (6.8) exists.

We shall frequently use the following lemma. We assume that $F: G^{\prime} \times R^{n} \rightarrow R$ satisfies (a)-(c) for $p=n$ in $G^{\prime}$.

6.9. Lemma. Suppose that $u$ is an $A C L^{n}$-function in $G^{\prime}$ and that $f: G \rightarrow G^{\prime}$ is qr. Then $v=u \circ$ fis $A C L^{n}$ in $G$ and

$$
\nabla v(x)=f^{\prime}(x)^{*} \nabla u(f(x))
$$

a.e. in G. Furthermore, if $D \subset \subset G$ is a normal domain of $f$, then

$$
\int_{D} F(f(x), \nabla u(f(x))) J(x, f) d m(x)=N(f, D) \int_{f D} F(y, \nabla u(y)) d m(y) .
$$

Proof. We may assume that $G$ is a domain and $f$ is nonconstant. Since $f$ is a.e. differentiable, (6.10) holds. Next we prove the formula (6.11). For this express $f D \backslash f\left(D \cap B_{f}\right), B_{f}$ being the branch set of $f$ [MRV1, p. 7], as a countable union of essentially disjoint closed cubes $Q_{i}$. Using then the fact that $m\left(B_{f}\right)=0=m\left(f B_{f}\right)$, see [MRV1, 8.3 and 2.27], and that there exist exactly $N(f, D)$-components of $f^{-1} Q_{i}$ in $D$, the formula (6.11) follows from (6.8).

For the rest of the proof observe that $v$ is clearly continuous. Since the $A C L^{n}$ property is local and every point of $G$ belongs to some normal domain $D$, it remains to show that $v \in W_{n}^{1}(D)$.

To this end, choose a sequence $u_{i} \in C^{\infty}\left(G^{\prime}\right)$ such that $u_{i} \rightarrow u$ uniformly in $f D$ and $\nabla u_{i} \rightarrow \nabla u$ in $L^{n}(f D)$. Set $v_{i}=u_{i} \circ f$. Then $v_{i}$ is clearly $A C L$ in $G$. Choose $F(y, h)=|h|^{n}$ in (6.11). By (6.10) and (6.11)

$$
\begin{aligned}
\int_{D}\left|\nabla v_{i}\right|^{n} d m & =\int_{D}\left|f^{\prime}(x)^{*} \nabla u_{i}(f(x))\right|^{n} d m(x) \\
& \leqslant K_{0}(f) \int_{D}\left|\nabla u_{i}(f(x))\right|^{n} J(x, f) d m(x) \\
& =K_{0}(f) N(f, D) \int_{f D}\left|\nabla u_{i}\right|^{n} d m
\end{aligned}
$$

where the quasiregularity of $f$ has also been used. This shows that the sequence $\left(\nabla v_{i}\right)$ is bounded in $L^{n}(D)$. Since $v_{i} \rightarrow v$ uniformly in $D, v \in W_{n}^{1}(D)$. Thus $v$ is $A C L^{n}$. This completes the proof.

The next lemma shows the invariance of variational integrals in the qc case. We assume that $F$ satisfies (a)-(c) and (e) for $p=n$ in $G^{\prime}$.

6.12. Lemma. Suppose that $u$ is an $A C L^{n}$-function on $G^{\prime}$ and that $f: G \rightarrow G^{\prime}$ is a qc homeomorphism. Then $I_{F}(u, A)=I_{f^{\ddagger} F}\left(u \circ f, f^{-1} A\right)$ for each measurable set $A \subset G^{\prime}$. 
Proof. Since $J(x, f)>0$ a.e. and $I_{F}(u, A)$ certainly exists, we may apply (6.8). Then by (6.10) and (e)

$$
\begin{aligned}
I_{f^{*} F}\left(u \circ f, f^{-1} A\right) & =\int_{f^{-1} A} F\left(f(x), J(x, f)^{1 / n} f^{\prime}(x)^{-1^{*}} f^{\prime}(x)^{*} \nabla u(f(x))\right) d m(x) \\
& =\int_{f^{-1} A} J(x, f) F(f(x), \nabla u(f(x))) d m(x)=I_{F}(u, A) .
\end{aligned}
$$

This proves the lemma.

In the qr case the following counterpart of Lemma 6.12 is adequate for our purposes.

6.13. LeMMA. Let $f: G \rightarrow G^{\prime}$ be a nonconstant qr mapping and $G \subset R^{n}$ a domain. If $D \subset \subset G$ is a normal domain of $f$, then $I_{f^{\mp} F}(u \circ f, D)=N(f, D) I_{F}(u, f D)$ for all $A C L^{n}$-functions $u$ in $G^{\prime}$.

Proof. By Lemma 6.9, $v=u \circ f$ is $A C L^{n}$ in $G$ and thus $I_{f^{*} F}(v, D)$ is finite. Since $J(x, f)>0$ a.e., see [MRV1, 8.2], the formulas (6.11) and (6.10) and the definition of $f^{\neq} F$ yield as in the proof of Lemma 6.12

$$
\begin{aligned}
I_{f^{\star} F}(v, D) & =\int_{D} J(x, f) F(f(x), \nabla u(f(x))) d m(x) \\
& =N(f, D) \int_{f D} F(y, \nabla u) d m(y)=N(f, D) I_{F}(u, f D) .
\end{aligned}
$$

This completes the proof.

7. Quasiregular mappings and $F$-extremals. Harmonic and subharmonic functions remain invariant under composition with analytic functions. This section is devoted to the corresponding invariance properties of $F$-extremals and sub- $F$-extremals with respect to quasiregular mappings. The case of $F$-extremals has been studied by $\mathrm{Yu}$. Rešetnjak [R4] in a special case, see Remark 3.4. However, our method is different.

We first consider the quasiconformal case.

7.1. THEOREM. Let $F: G^{\prime} \times R^{n} \rightarrow R$ be a variational kernel satisfying (a)-(c) in an open set $G^{\prime} \subset R^{n}$ for $p=n$. If $f: G \rightarrow G^{\prime}$ is a qc homeomorphism, then a function $u$ in $G^{\prime}$ is a sub-F-extremal, a regular sub-F-extremal or an F-extremal if and only if $u \circ f$ is a corresponding $f^{\neq}$F-extremal in $G$.

Proof. For a.e. $y \in G^{\prime}, f^{-1^{*}} f^{\neq} F(y, h)=F(y, h)$ for all $h \in R^{n}$. Thus it suffices to prove the only if part. Suppose first that $u: G^{\prime} \rightarrow R$ is an $F$-extremal. Let $D \subset \subset G$ and set $D^{\prime}=f D$. Fix $v \in C(\bar{D}) \cap W_{n}^{l}(D)$ with $v|\partial D=u \circ f| \partial D$. An application of Lemma 6.12 twice and the extremality of $u$ in $D^{\prime}$ yield

$$
\begin{aligned}
I_{f^{\ddagger} F}(u \circ f, D) & =I_{F}\left(u, D^{\prime}\right) \leqslant I_{F}\left(v \circ f^{-1}, D^{\prime}\right) \\
& =I_{f^{\ddagger} F}\left(v \circ f^{-1} \circ f, D\right)=I_{f^{\mp} F}(v, D) .
\end{aligned}
$$

This shows that $u \circ f$ is an $f^{\neq} F$-extremal in $G$.

Suppose next that $u: G^{\prime} \rightarrow R \cup\{-\infty\}$ is a sub- $F$-extremal. Let again $D \subset \subset G$ be a domain and $D^{\prime}=f D$. If $h \in C(\bar{D})$ is an $f^{\neq} F$-extremal in $D$ with $h \geqslant u \circ f$ in $\partial D$, 
then, as we just have seen, $h \circ f^{-1}$ is an $F$-extremal in $D^{\prime}$. On the other hand, $h \circ f^{-1} \geqslant u$ in $\partial D^{\prime}$ and since $u$ is a sub-F-extremal, $h \circ f^{-1} \geqslant u$ in $D^{\prime}$. This proves $h \geqslant u \circ f$ in $D$ and since $u \circ f$ is clearly upper semicontinuous, $u \circ f$ is a sub- $F$ extremal in $G$.

In view of the above considerations the case of regular subextremals is clear, since if $u$ is $A C L^{n}$ in $G^{\prime}$, then by Lemma 6.9, $u \circ f$ is $A C L^{n}$ in $G$. The proof is complete.

7.2. Quasiregular mappings. It is well known that if $f: G \rightarrow G^{\prime}$ is analytic from an open set $G \subset R^{2}$ into an open set $G^{\prime} \subset R^{2}$, then $u \circ f$ is harmonic or $C^{2}$-subharmonic in $G$ whenever $u$ is a similar function in $G^{\prime}$ and the proof for these two facts is elementary. If $u$ is subharmonic, then $u \circ f$ is also subharmonic. The proof usually employs the submean property of subharmonic functions and the local behavior of analytic functions at a point, cf. [HK, p. 53]. Here we prove that all classes of $F$-extremals remain invariant under composition of quasiregular mappings.

To study the above problems a certain "lifting" of functions defined on $G$ to $G^{\prime}$ is needed. These studies have their origin in [M1].

Suppose that $G$ is a domain and $G^{\prime}$ an open set in $R^{n}$ and $f: G \rightarrow G^{\prime}$ is a nonconstant qr mapping. Let $D$ be a normal domain of $f$ and let $v: D \rightarrow R$ be a function. For $y \in f D$ set

$$
v^{*}(y)=\frac{1}{N(f, D)} \sum_{x \in f^{-1}(y) \cap D} i(x, f) v(x) .
$$

In the following three lemmas we study the properties of the function $v^{*}$. We assume that $F: G^{\prime} \times R^{n} \rightarrow R$ satisfies the assumptions (a)-(c) and (e) for $p=n$.

7.4. Lemma. Suppose that $v \in C(D) \cap W_{n}^{1}(D)$. Then $v^{*}$ belongs to $C(f D) \cap$ $W_{n}^{1}(f D)$ and

$$
N(f, D) I_{F}\left(v^{*}, f D\right) \leqslant I_{f^{*} F}(v, D)
$$

Proof. We first show that $v^{*}$ is continuous on $f D$. Fix $y \in f D$. Let $\varepsilon>0$. Then the continuity of $v$ and [MRV1, 2.9 and 2.10] show that there is $r \in(0, \operatorname{dist}(y, \partial f D))$ such that for all $x \in f^{-1}(y) \cap D$ the $x$-component $U(x, f, r)$ of $f^{-1} B^{n}(y, r)$ is a normal neighborhood of $x$ and $\left|v\left(x^{\prime}\right)-v(x)\right|<\varepsilon$ for all $x^{\prime} \in U(x, f, r)$. Fix such an $r$. Set $U_{i}=U\left(x_{i}, f, r\right)$ for $x_{i} \in f^{-1}(y) \cap D, i=1,2, \ldots, k$, and $U=\cup U_{i}$. Let $y^{\prime} \in B^{n}(y, r)$. Then $f^{-1}\left(y^{\prime}\right) \cap D \subset U$ and

$$
\begin{array}{r}
\left|v^{*}\left(y^{\prime}\right)-v^{*}(y)\right|=\left|\frac{1}{N(f, D)}\left[\sum_{x^{\prime} \in f^{-1}\left(y^{\prime}\right) \cap D} i\left(x^{\prime}, f\right) v\left(x^{\prime}\right)-\sum_{i=1}^{k} i\left(x_{i}, f\right) v\left(x_{i}\right)\right]\right| \\
=\frac{1}{N(f, D)}\left|\sum_{i=1}^{k}\left(\sum_{x^{\prime} \in f^{-1}\left(y^{\prime}\right) \cap U_{i}} i\left(x^{\prime}, f\right) v\left(x^{\prime}\right)-i\left(x_{i}, f\right) v\left(x_{i}\right)\right)\right|<\varepsilon,
\end{array}
$$

since $\sum_{x^{\prime} \in f^{-1}\left(y^{\prime}\right) \cap U_{i}} i\left(x^{\prime}, f\right)=i\left(x_{i}, f\right)$ and $\sum_{i=1}^{k} i\left(x_{i}, f\right)=N(f, D)$, see 6.1. This proves the continuity of $v^{*}$. 
Suppose for a moment that $v^{*}$ is $A C L$ in $f D$. We show that (7.5) holds in this case. For this we follow [M1]. Since $m\left(f B_{f}\right)=0$, we may express the open set

$$
f D \backslash f\left(B_{f} \cap D\right)
$$

as a union of essentially disjoint closed cubes $Q_{i}, i=1,2, \ldots$, and $m\left(f D \backslash \cup Q_{i}\right)=$ 0 . Set $k=N(f, D)$ and $Q_{i}^{0}=$ int $Q_{i}$. Then each $D \cap f^{-1} Q_{i}^{0}$ consists of $k$ disjoint domains $D_{i}^{j}, j=1,2, \ldots, k$, and $D_{i}^{j}$ is mapped quasiconformally by $f$ onto $Q_{i}^{0}$. It suffices to show

$$
k I_{F}\left(v^{*}, Q_{i}^{0}\right) \leqslant I_{f^{*} F}\left(v, \bigcup_{j=1}^{k} D_{i}^{j}\right) .
$$

To this end fix $i$ and set $g_{j}=\left(f \mid D_{i}^{j}\right)^{-1}$. For $y \in Q_{i}^{0}, v^{*}(y)=\frac{1}{k} \sum_{j=1}^{k} v\left(g_{j}(y)\right)$ and $\nabla v^{*}(y)=\frac{1}{k} \sum_{j=1}^{k} \nabla\left(v \circ g_{j}\right)(y)$ a.e. in $Q_{i}^{0}$. Since $F$ is convex,

$$
F\left(y, \nabla v^{*}(y)\right) \leqslant \frac{1}{k} \sum_{j=1}^{k} F\left(y, \nabla\left(v \circ g_{j}\right)(y)\right)
$$

a.e. in $Q_{i}^{0}$. Thus by Lemma 6.12

$$
\begin{aligned}
k I_{F}\left(v^{*}, Q_{i}^{0}\right) & \leqslant \sum_{j=1}^{k} I_{F}\left(v \circ g_{j}, Q_{i}^{0}\right)=\sum_{j=1}^{k} I_{g_{j}^{-1} F_{F}}\left(v \circ g_{j} \circ g_{j}^{-1}, D_{i}^{j}\right) \\
& =\sum_{j=1}^{k} I_{f^{\ddagger} F}\left(v, D_{i}^{j}\right) .
\end{aligned}
$$

This proves (7.6). Since

$$
\alpha(F) \int_{f D}\left|\nabla v^{*}\right|^{n} d m \leqslant I_{F}\left(v^{*}, f D\right),
$$

(7.5) and Lemma 6.4 imply that $v^{*} \in W_{n}^{1}(f D)$.

If $v \in C^{\infty}(D)$, then the proof of [M1, Lemma 5.5] shows that $v^{*}$ is $A C L$ in $f D$. Suppose now that $v \in C(D) \cap W_{n}^{1}(D)$. Then there exists a sequence $v_{i} \in C^{\infty}(D)$ such that $v_{i} \rightarrow v$ locally uniformly in $D$ and $\nabla v_{i} \rightarrow \nabla v$ in $L^{n}(D)$. Since for each $y \in f D$

$$
\left|v_{i}^{*}(y)-v^{*}(y)\right| \leqslant \sup _{x \in f^{-1}(y) \cap D}\left|v_{i}(x)-v(x)\right|,
$$

$v_{i}^{*} \rightarrow v^{*}$ locally uniformly in $f D$. On the other hand we have just shown that (7.5) holds for $v_{i}$, hence by Lemma 6.4

$$
\begin{aligned}
\alpha(F) \int_{f D}\left|\nabla v_{i}^{*}\right|^{n} d m & \leqslant I_{F}\left(v_{i}^{*}, f D\right) \leqslant \frac{1}{N(f, D)} I_{f^{\ddagger} F}\left(v_{i}, D\right) \\
& \leqslant \frac{\beta(F) K_{I}(f)}{N(f, D)} \int_{D}\left|\nabla v_{i}\right|^{n} d m .
\end{aligned}
$$

Since the sequence $\left\|\nabla v_{i}\right\|_{n}$ is bounded, passing to a subsequence, if necessary, $\nabla v_{i}^{*}$ converges weakly in $L^{n}(f D)$ to $\nabla v^{*}$. Consequently, $v^{*} \in W_{n}^{1}(f D)$. But if $v^{*} \in$ $C(f D) \cap W_{n}^{1}(f D)$, then we have showed that (7.5) holds. This completes the proof. 
7.7. LemMA. Suppose that $v \in C(\bar{D})$ and $v=u \circ f$ on $\partial D$ for some $u \in C(\partial f D)$. Then $v^{*}$ has a continuous extension to $\partial f D$, denoted again by $v^{*}$, and $v^{*}=u$ on $\partial f D$.

Proof. By Tietze's extension theorem we may assume that $u \in C\left(R^{n}\right)$. Let $\left(y_{i}\right)$ be a sequence of points in $f D$ with $y_{i} \rightarrow y_{0} \in \partial f D$. Using the fact that $f$ is discrete and open and that $D$ is a normal domain of $f$, it is easy to see that for each $\varepsilon>0$ there is $\delta>0$ such that for $E \subset f D, \operatorname{dist}(E, \partial f D)<\delta$ implies $\operatorname{dist}\left(f^{-1} E \cap D, \partial D\right)<\varepsilon$. Let $\varepsilon>0$. Since $v=u \circ f$ on $\partial D$ and $u$ and $v$ are continuous, it is possible to apply the above fact to the set $E_{i}=\left\{y_{i}, y_{i+1}, \ldots\right\}$ and obtain $i_{0}$ such that $|v(x)-u(f(x))|<\varepsilon$ for $x \in f^{-1}\left(E_{i_{0}}\right) \cap D$. Then for $i \geqslant i_{0}$

$$
\begin{aligned}
\left|v^{*}\left(y_{i}\right)-u\left(y_{0}\right)\right| \leqslant\left|v^{*}\left(y_{i}\right)-u\left(y_{i}\right)\right|+\left|u\left(y_{i}\right)-u\left(y_{0}\right)\right| \\
\quad=\left|\frac{1}{N(f, D)} \sum_{x \in f^{-1}\left(y_{i}\right) \cap D} i(x, f)(v(x)-u(f(x)))\right|+\left|u\left(y_{i}\right)-u\left(y_{0}\right)\right| \\
\quad<\varepsilon+\left|u\left(y_{i}\right)-u\left(y_{0}\right)\right| .
\end{aligned}
$$

Thus $\lim v^{*}\left(y_{i}\right)=u\left(y_{0}\right)$ and the lemma follows.

7.8. Remark. If $D$ is a normal domain for $f$, then it is possible to define $v_{1}$ : $\overline{f D} \rightarrow R$ using the formula (7.3) in $\overline{f D}$. However, this $v_{1}$ is not always continuous in $\overline{f D}$. To see this consider the plane analytic function $f(z)=z^{2}$ and a normal domain $D$ of $f$ such that $f D=B^{2}(1,1)$. Let $v(x)=1$ for all $x \in R^{2}$. Then $v_{1}(0)=2 \neq 1=$ $\lim _{y \rightarrow 0} v_{1}(y), y \in f D$. Observe that if $v$ has compact support in $D$, so has $v^{*}$ in $f D$ and the above situation cannot occur.

7.9. Lemma. Suppose that $v \in C(\bar{D}) \cap W_{n}^{1}(D)$ and $v$ is an $f^{\neq} F$-extremal in $D$. If $v=u \circ f$ for some $u \in C(f \partial D)$, then $v^{*}$ is an F-extremal in $f D$.

Proof. Let $w \in C(\overline{f D}) \cap W_{n}^{1}(f D)$ be such that $w=v^{*}$ on $\partial f D$. By Lemma 7.7, $w \circ f=v^{*} \circ f=u \circ f=v$ in $\partial D$, thus Lemma 6.13, the extremality of $v$, and Lemma 7.4 yield

$$
N(f, D) I_{F}(w, f D)=I_{f^{\neq} F}(w \circ f, D) \geqslant I_{f^{*} F}(v, D) \geqslant N(f, D) I_{F}\left(v^{*}, f D\right) .
$$

Hence $I_{F}(w, f D) \geqslant I_{F}\left(v^{*}, f D\right)$. This completes the proof.

7.10. Theorem. Suppose that $f: G \rightarrow G^{\prime}$ is quasiregular and $F: G^{\prime} \times R^{n} \rightarrow R$ satisfies the assumptions (a), (b'), (c)-(e) in $G^{\prime}$. If $u$ is an F-extremal, a regular sub-F-extremal or a sub-F-extremal in $G^{\prime}$, then $u \circ f$ is a corresponding $f^{\neq} F$-extremal in $G$.

Proof. By Lemma 6.9 it suffices to consider the cases: (i) $u$ is an $F$-extremal and (ii) $u$ is a sub- $F$-extremal. Clearly we may assume that $G$ is a domain and $f$ is nonconstant.

Case (i). Let $u$ be an $F$-extremal in $G^{\prime}$. Set $v=u \circ f$. Then, by Lemma 6.9, $v$ is $A C L^{n}$ in $G$. Since the $f^{\neq} F$-extremality is a local property, see Remark 3.28 , it suffices to show that $v$ is an $f^{\neq} F$-extremal in a normal neighborhood $U=U\left(x_{0}, f, r\right)$ 
of $x_{0} \in G$. Since a normal neighborhood is a normal domain, we may apply the previous lemmas. Let $w \in C(\bar{U}) \cap W_{n}^{1}(U)$ with $w=v$ on $\partial U$. Consider $w^{*}: \overline{f U} \rightarrow R$. By Lemma $7.7 w^{*}=u$ on $\partial f U$ and by the extremality of $u$, Lemma 7.4, and Lemma 6.13

$$
I_{f^{\ddagger} F}(w, U) \geqslant N(f, U) I_{F}\left(w^{*}, f U\right) \geqslant N(f, U) I_{F}(u, f U)=I_{f^{\ddagger} F}(v, U),
$$

which proves the $f^{\neq} F$-extremality of $v$.

Case (ii). Let $u$ be a sub- $F$-extremal in $G^{\prime}$. Set $v=u \circ f$. First we show that $v$ satisfies the $f^{\neq} F$-comparison principle, see 5.22, in each regular normal domain $D$ of $f$.

Let $D$ be a regular normal domain of $f$ and $\varepsilon>0$. Let $h \in C(\bar{D}) \cap W_{n}^{1}(D)$ be an $f^{\neq} F$-extremal in $D$ with $h \geqslant v+\varepsilon$ in $\partial D$. There is a function $\psi \in C(\overline{f D}) \cap W_{n}^{1}(f D)$ with $h \geqslant \psi \circ f$ in $\partial D$ and $\psi \geqslant u$ in $\partial f D$. Let $h_{1}$ be the $f^{\neq} F$-extremal in $D$ with boundary values $\psi \circ f$ in $\partial D$. Then $h \geqslant h_{1}$ in $D$ and it remains to show $h_{1} \geqslant v$ in $D$.

To this end observe that by Lemmas 7.7 and 7.9, $h_{1}^{*}=\psi$ on $\partial f D$ and $h_{1}^{*}$ is an $F$-extremal in $f D$. Thus $h_{1}^{*} \geqslant u$ in $f D$ and by the uniqueness of $f^{\neq} F$-extremals, $h_{1}=h_{1}^{*} \circ f$. Hence $h_{1}=h_{1}^{*} \circ f \geqslant u \circ f=v$ in $D$. We have shown that $v$ satisfies the comparison principle in $D$.

By Lemma 5.23 it now suffices to show that $G$ has a regular base consisting only of regular normal domains. In $G^{\prime}$ we first introduce a simple regular base $\mathfrak{B}^{\prime}$. Consider for $j=1,2, \ldots$ the standard subdivision of $R^{n}$ into open disjoint cubes $Q$ parallel to the coordinate axes and side length $2^{-j}$. For each such $Q$ let $Q^{\prime}$ denote the enlargement of $Q$ with side length $52^{-j} / 4$ and with the same center. Let $A_{j}$ be the set of all cubes $Q^{\prime}$ in $G^{\prime}$. Then $\mathfrak{B}^{\prime}=\cup A_{j}$ is a regular base in $G^{\prime}$ and it has the following property: If $D^{\prime} \subset G^{\prime}$ is a domain and $K^{\prime}$ compact in $D^{\prime}$, then there is $j_{0}$ such that for $j \geqslant j_{0}$ the finite collection $\left\{Q^{\prime} \in A_{j}: Q^{\prime} \cap K^{\prime} \neq \varnothing\right\}$ is a regular cover of $K^{\prime}$ in $D^{\prime}$.

In $G$ we proceed as follows. Let $\mathscr{B}$ be the family of all normal domains $D$ in $G$ such that $D$ is a component of $f^{-1} Q^{\prime}$ for some $Q^{\prime} \in \mathscr{B}^{\prime}$. We show that $\mathscr{B}$ is a regular base for $G$. Let $V \subset G$ be a domain and $K$ compact in $V$. It is easy to see that there is $j_{0}$ such that for $j \geqslant j_{0}$ the finite collection

$$
C_{j}=\left\{D \in \mathscr{B}: f D=Q^{\prime}, Q^{\prime} \in A_{j}, D \cap K \neq \varnothing\right\}
$$

covers $K$ and $\cup C_{j} \subset V$. Let $j=j_{0}+1$ and $C_{j}=\left\{D_{1}, \ldots, D_{k}\right\}$. If $U$ is a union of some subfamily of $C_{j}$ 's, say $U=\bigcup_{i=1}^{p} D_{i}, 1 \leqslant p \leqslant k$, it remains to show that $\partial U$ is without point components.

To this end let $x_{0} \in \partial U$. We may assume that $D_{1}, \ldots, D_{l}, 1 \leqslant l \leqslant p$, are those $D_{i}$ 's whose closure contains $x_{0}$. Write $Q_{i}^{\prime}=f D_{i}, 1 \leqslant i \leqslant l$, and $P=\cup_{i=1}^{l} Q_{i}^{\prime}$. Then $f\left(x_{0}\right) \in \partial P$ and there is at least one cube $Q^{\prime} \in A_{j}$ such that $f\left(x_{0}\right) \in Q^{\prime}$ and $Q^{\prime} \neq Q_{i}^{\prime}$ for $i=1, \ldots, l$. Choose a nondegenerate curve $\gamma$ starting at $f\left(x_{0}\right)$ inside $Q^{\prime} \backslash P$. A lift $\gamma^{*}$ of $\gamma$ starting at $x_{0}$, see [MRV1, Lemma 2.7], is inside the $x_{0}$-component of $f^{-1} Q^{\prime}$, which is a normal domain of $f$. On the other hand, $\gamma^{*}$ is outside $\cup_{i=1}^{l} D_{i}$. Thus at least a short piece of $\gamma^{*}$ near $x_{0}$ is outside $U$. Hence $\left\{x_{0}\right\}$ is not a point component of $\partial U$. This completes the proof. 


\section{REFERENCES}

[GZ] R. Gariepy and W. P. Ziemer, A regularity condition at the boundary for solutions of quasilinear elliptic equations, Arch. Rational Mech. Anal. 67 (1977), 25-39.

[G] F. W. Gehring, Rings and quasiconformal mappings in space, Trans. Amer. Math. Soc. 103 (1962), 353-393.

[Gr] S. Granlund, Harnack's inequality in the borderline case, Ann. Acad. Sci. Fenn. Ser. A I Math. 5 (1980), 159-164.

[HK] W. K. Hayman and P. B. Kennedy, Subharmonic functions, Academic Press, New York, 1976.

[L] H. Lebesgue, Sur le problème de Dirichlet, Rend. Circ. Mat. Palermo 24 (1907), 371-402.

[Li1] P. Lindqvist, $A$ new proof of the lower-semicontinuity of certain convex variational integrals in Sobolev spaces, Report HTKK-MAT-A97 (1977), 1-10.

[Li2] $117-122$. , On the Hölder continuity of monotone extremals in the borderline case, Ark. Mat. 19 (1981),

[M1] O. Martio, A capacity inequality for quasiregular mappings, Ann. Acad. Sci. Fenn. Ser. A I Math. 474 (1970), 1-18.

[M2] _ Equicontinuity theorem with an application to variational integrals, Duke Math. J. 42 (1975), 569-581.

[M3] _ Reflection principle for solutions of elliptic partial differential equations and quasiregular mappings, Ann. Acad. Sci. Fenn. Ser. A I Math. 7 (1981), 179-187.

[MRV1] O. Martio, S. Rickman and J. Väisälä, Definitions for quasiregular mappings, Ann. Acad. Sci. Fenn. Ser. A I Math. 448 (1969), 1-40.

[MRV2] _ Distortion and singularities of quasiregular mappings, Ann. Acad. Sci. Fenn. Ser. A I Math. 465 (1970), 1-13.

[MS] O. Martio and J. Sarvas, Density conditions in the n-capacity, Indiana Univ. Math. J. 26 (1977), 761-776.

[Maz] V. Mazja, On the continuity at a boundary point of the solution of quasi-linear elliptic equations, Vestnik Leningrad. Univ. Math. 25 (13) (1970), 42-55. (Russian)

[ME] N. G. Meyers and A. Elcrat, Some results on regularity for solutions of non-linear elliptic systems and quasiregular functions, Duke Math. J. 42 (1975), 121-136.

[Mor] C. B. Morrey, Multiple integrals in the calculus of variations, Springer-Verlag, Berlin and New York, 1966.

[Mos] G. D. Mostow, Quasiconformal mappings in n-space and the rigidity of hyperbolic space forms, Inst. Hautes Études Sci. Publ. Math. 34 (1968), 53-104.

[R1] Yu. G. Rešetnjak, Spatial mappings with bounded excursions, Sibirsk. Mat. Ž. 8 (1967), 629-658. (Russian)

[R2] _ General theorems on semicontinuity and on convergence with functionals, Sibirsk. Mat. Ž. 8 (1967), 1051-1069. (Russian)

[R3] _ Mappings with bounded deformation as extremals of Dirichlet type integrals, Sibirsk. Mat. $\check{Z}$. 9 (1968), 652-666. (Russian)

[R4] _ Extremal properties of mappings with bounded distortion, Sibirsk. Mat. Ž. 10 (1969), 1300-1310. (Russian)

[Ro] R. T. Rockafeller, Convex analysis, Princeton Univ. Press, Princeton, N. J., 1972.

[RR] T. Rado and P. V. Reichelderfer, Continuous transformations in analysis, Springer-Verlag, Berlin and New York, 1955.

[S] J. Serrin, Local behavior of solutions of quasilinear equations, Acta Math. 111 (1964), 247-302.

[V] J. Väisälä, Lectures on n-dimensional quasiconformal mappings, Lecture Notes in Math., vol. 229, Springer-Verlag, Berlin and New York, 1971.

Institute of Mathematics, Helsinki University of TeChNology, SF-02150 Espoo 15, Finland

DEPaRTMENT OF Mathematics, UNiverSity OF JYVÄSKYLÄ, SF-40100 JYVÄSKYLÅ 10, FinLAND 